\title{
The effect of the centrifugal acceleration on period spacings of gravito-inertial modes in intermediate-mass stars
}

\author{
J. Henneco ${ }^{1}$, T. Van Reeth ${ }^{1}$, V. Prat ${ }^{2}$, S. Mathis ${ }^{2}$, J. S. G. Mombarg ${ }^{1}$, and C. Aerts ${ }^{1,3,4}$ \\ 1 Institute of Astronomy, KU Leuven, Celestijnenlaan 200D, 3001 Leuven, Belgium \\ e-mail: jan.henneco@protonmail.com \\ 2 AIM, CEA, CNRS, Université Paris-Saclay, Université Paris Diderot, Sorbonne Paris Cité, 91191 Gif-sur-Yvette, France \\ 3 Department of Astrophysics, IMAPP, Radboud University Nijmegen, PO Box 9010, 6500 GL Nijmegen, the Netherlands \\ ${ }^{4}$ Max Planck Institute for Astronomy, Koenigstuhl 17, 69117 Heidelberg, Germany
}

January 13, 2021

\begin{abstract}
Context. The Kepler and TESS space telescopes delivered high-precision, long-duration photometric time series for hundreds of mainsequence stars, revealing their numerous gravito-inertial $(g)$ pulsation modes. This high precision allows us to evaluate increasingly detailed theoretical stellar models. Recent theoretical work extended the traditional approximation of rotation, a framework to evaluate the effect of the Coriolis acceleration on g-modes, to include the effects of the centrifugal acceleration in the approximation of slightly deformed stars, which so far had mostly been neglected in asteroseismology. This extension of the traditional approximation was conceived by rederiving the traditional approximation in a centrifugally deformed, spheroidal coordinate system.

Aims. We explore the effect of the centrifugal acceleration on g modes and assess its detectability in space-based photometric observations.

Methods. We implement the new theoretical framework to calculate the centrifugal deformation of precomputed 1D spherical stellar structure models and compute the corresponding g-mode frequencies, assuming uniform rotation. The framework is evaluated for a grid of stellar structure models covering a relevant parameter space for observed g-mode pulsators.

Results. The centrifugal acceleration modifies the effect of the Coriolis acceleration on $\mathrm{g}$ modes, narrowing the equatorial band in which they are trapped. Furthermore, the centrifugal acceleration causes the pulsation periods and period spacings of the most common g modes (prograde dipole modes and $\mathrm{r}$ modes) to increase with values similar to the observational uncertainties of the measured period spacing values in Kepler and TESS data.

Conclusions. The effect of the centrifugal acceleration on g modes is formally detectable in modern space photometry. Implementation of the used theoretical framework in stellar structure and pulsation codes will allow for more precise asteroseismic modelling of centrifugally deformed stars, in order to assess its effect on mode excitation, -trapping and -damping.
\end{abstract}

Key words. asteroseismology - waves - stars: oscillations - stars: rotation - stars: interiors - hydrodynamics

\section{Introduction}

Over the last decade, there have been major advancements in observational asteroseismology. Thanks to space missions such as CoRoT (Convection, Rotation and planetary Transits; Auvergne et al. 2009), Kepler (Borucki et al. 2009), the BRITEConstellation (BRIght Target Explorer Constellation; Weiss et al. 2014) and TESS (Transiting Exoplanet Survey Satellite; Ricker et al. 2014), long-time-base, high-cadence, highprecision photometric light curves are now available for hundreds of thousands of stars. This has resulted in the detection and identification of g-mode oscillations, which have buoyancy as the main restoring force, in hundreds of $\gamma$ Doradus ( $\gamma$ Dor) and slowly-pulsating B-type (SPB) stars (e.g., Tkachenko et al. 2013; Van Reeth et al. 2015; Pápics et al. 2017; Christophe et al 2018; Li et al. 2019b,c, 2020, Pedersen et al., submitted).

$\gamma$ Dor (Kaye et al. 1999) and SPB stars (Waelkens 1991) are main-sequence stars, with masses $1.4 \mathrm{M}_{\odot} \lesssim M \lesssim 1.9 \mathrm{M}_{\odot}$ and $3 \mathrm{M}_{\odot} \lesssim M \lesssim 9 \mathrm{M}_{\odot}$, respectively. Their g-mode pulsations have periods between 0.3 and 5 days, and are mostly sensitive to the near-core regions of the stars. Asteroseismic modelling of observed and identified g-mode pulsations allows us to constrain the physical processes taking place in the deep stellar interior (see e.g. Aerts et al. 2018). While similar on some fronts, major differences occur in the modelling of $\mathrm{g}$ modes compared to the case of stochastically-excited pressure modes (see Aerts 2021, for an extensive review on the overall methodology). For $\mathrm{g}$ modes in $\gamma$ Dor and SPB stars, key processes to infer are convective core overshooting or convective penetration in the core boundary layers (e.g., Pedersen et al. 2018; Michielsen et al. 2019), interior magnetic fields (Prat et al. 2019, 2020; Van Beeck et al. 2020; Mathis et al. 2021, Bugnet et al. submitted), and microscopic or macroscopic mixing in the radiative envelope (e.g., Deal et al. 2016; Rogers \& McElwaine 2017; Pedersen et al. 2018; Mombarg et al. 2020; Mathis et al. 2021, Pedersen et al., submitted; Bugnet et al., submitted).

One of the most crucial aspects that have to be taken into account in stellar structure and evolution theory is rotation (Zahn 1992; Maeder \& Zahn 1998; Mathis \& Zahn 2004; Maeder 2009, and references therein). Aside from causing numerous physical processes such as rotational mixing, stellar rotation severely influences the behaviour of g-mode pulsations via the Coriolis acceleration (e.g., Lee \& Saio 1997; Dintrans \& Rieutord 2000; Aerts et al. 2019, for a review). For high-frequency oscillations $(\omega \gg \Omega$, with $\omega$ the angular pulsation frequency in 
the co-rotating frame and $\Omega$ the angular rotation rate) the Coriolis acceleration can be treated as a perturbation (Hansen et al. 1977; Gough 1981). However, high-order g modes (with radial order $n \gg$ spherical degree $\ell$ ) typically lie in the low-frequency range $(\omega \lesssim \Omega$ ), where the Coriolis force also contributes to the restoring of the oscillations. Such g-mode pulsations therefore occur in the gravito-inertial regime and correspond to gravitoinertial waves (GIW, hereafter). Hence, the Coriolis force can no longer be treated as a perturbation, and the hydrodynamical equations that govern the oscillations become an infinite set of coupled differential equations (Mathis 2009). However, approximate numerical solutions and general properties of GIW can still be obtained by truncating the infinite set of coupled differential equations (e.g., Berthomieu et al. 1978; Lee \& Saio 1986, 1987; Dziembowski \& Kosovichev 1987b,a; Dziembowski et al. 1987; Dintrans et al. 1999; Dintrans \& Rieutord 2000; Mathis 2009).

The influence of the Coriolis force on GIWs is commonly described using the traditional approximation of rotation (TAR). It was first developed by Eckart (1960) in his study of the dynamics of shallow atmospheres and oceans on Earth, and later introduced in stellar pulsation theory by Berthomieu et al. (1978) and Lee \& Saio (1987). The main assumption of the TAR is that the stratification in which the waves propagate, is sufficiently strong as to limit vertical wave motions. As a consequence, the horizontal component of the rotation vector and therefore also the vertical component of the Coriolis acceleration, can be neglected within the description of the GIWs. This condition for stable stratification (both in chemical composition and in entropy) is typically met in the radiative near-core region of $\gamma$ Dor and SPB stars. By applying the TAR, the hydrodynamical oscillation equations can be decoupled and rewritten in the form of the Laplace tidal equation (Laplace 1799).

Two additional assumptions made within the TAR are those of uniform rotation and spherical symmetry. Mathis (2009) abandoned the first assumption and included the effect of differential rotation within the framework of the TAR. Subsequently, the sensitivity of GIWs to the effect of differential rotation was assessed by Van Reeth et al. (2018). The assumption of spherical symmetry is valid when the star is rotating sufficiently slowly to ignore the centrifugal acceleration, i.e., $\Omega \ll \Omega_{\mathrm{c}}$, where $\Omega_{\mathrm{c}}=\sqrt{G M_{\star} / R_{\mathrm{eq}}^{3}}=\sqrt{8 G M_{\star} / 27 R_{\text {pole }}^{3}} \simeq \sqrt{8 G M_{\star} / 27 R^{3}}$ is the Roche critical rotation rate, $G$ the universal gravitational constant and where $R_{\text {eq }}$ and $R_{\text {pole }}$ stand for the equatorial and polar radius of the star, respectively (see Maeder 2009, Chapter 2). However, a significant fraction of the $\gamma$ Dor and SPB stars are moderate to fast rotators (Pápics et al. 2017; Li et al. 2020, Pedersen et al., submitted). Hence, the effect of the centrifugal deformation should be taken into account in the theoretical description of their g-mode pulsations.

Mathis \& Prat (2019) generalised the TAR for moderatelyto-rapidly rotating stars by considering the effects of the centrifugal acceleration. First, they provide a prescription to deform a stellar structure model into a centrifugally deformed oblate spheroid. From the resulting perturbed physical quantities, a dimensionless deformation factor proportional to the square of the rotation rate can then be calculated. Secondly, this deformation factor is used to transition from a spherically symmetric to a spheroidal coordinate system, keeping only first-order terms in the deformation. Next, they re-derive the Laplace tidal equation within this new coordinate system, arriving at the so-called generalised Laplace tidal equation. Finally, the authors derived an asymptotic expression for the frequencies of GIWs, including the effect of the centrifugal acceleration.
In this work, we have set up a parameter study with the goal of assessing the effect of the centrifugal acceleration on g-mode pulsations in rotationally deformed stars and their detectability in space-based photometric observations. By doing so, we expand upon the proof-of-concept study conducted in the theoretical work of Mathis \& Prat (2019) and aim to answer the question whether the effect of the centrifugal acceleration should be accounted for in asteroseimic modelling of observed g-mode pulsators. A brief summary of the theoretical results and numerical implementation by Mathis \& Prat (2019), as well as our improvements to the theoretical framework, are provided in Sect. 2. The results of our upgraded implementation and parameter study follows in Sect. 3. We discuss the results and conclude in Sect. 4.

\section{Methodology}

\subsection{Theoretical background}

\subsubsection{Deformation of stellar structure}

Following Mathis \& Prat (2019, Appendix A), the pressure $P$, density $\rho$ and gravitational potential $\phi$ in a centrifugally deformed star are written as the sum of a spherically symmetric, non-perturbed part (subscript ' 0 ') and a perturbation (subscript ' 1 '). This perturbation term itself is then expanded on an orthogonal basis of Legendre polynomials $P_{\operatorname{leg}, l}(\cos \theta)$ of degree $l=0,2$, with $\theta$ the co-latitude and $\theta=0$ on the rotation axis. Such an expansion corresponds to the projection of the perturbed quantities on a spheroidal surface in the moderately rapid rotation regime considered here. For fast rotators, additional terms of (even) degree $l$ should be included but that is beyond our current scope.

For the gravitational potential, with $\phi_{0}=-G M(r) / r$ and $M(r)$ the mass contained within a sphere of radius $r$, we have:

$\phi(r, \theta)=\phi_{0}(r)+\phi_{1}(r, \theta)=\phi_{0}(r)+\sum_{l=0,2} \phi_{l}(r) P_{\operatorname{leg}, l}(\cos \theta)$.

With the inclusion of the centrifugal acceleration, the hydrostatic equilibrium in the star is written as:

$\frac{\boldsymbol{\nabla} P}{\rho}=-\boldsymbol{\nabla} \phi+\frac{1}{2} \Omega^{2} \boldsymbol{\nabla}\left(r^{2} \sin ^{2} \theta\right)$.

This last term corresponds to the gradient of the centrifugal potential (per unit mass) $U(r, \theta)=-\frac{1}{2} \Omega^{2} r^{2} \sin ^{2} \theta$. The centrifugal potential itself is then also expanded on the same basis of Legendre polynomials as:

$U=\sum_{l=0,2} U_{l}(r) P_{\operatorname{leg}, l}(\cos \theta)$.

This implies that the modal amplitudes of the centrifugal potential are given by $U_{l=0}=-(1 / 3) \Omega^{2} r^{2}$ and $U_{l=2}=(1 / 3) \Omega^{2} r^{2}$.

The modal amplitudes of the pressure and density, $P_{l}(r)$ and $\rho_{l}(r)$, are recovered from the property that in the centrifugally deformed star the equipotential surface $(\phi+U)$, isobar surface and isodensity surface coincide and are given by (Mathis \& Prat 2019, Appendix A):

$P_{l}(r)=-\rho_{0}(r)\left[\phi_{l}(r)+U_{l}(r)\right]$,

$\rho_{l}(r)=\frac{1}{g_{0}(r)} \frac{\mathrm{d} \rho_{0}(\mathrm{r})}{\mathrm{dr}}\left[\phi_{l}(r)+U_{l}(r)\right]$.

For the modal amplitude of the gravitational potential, $\phi_{l}(r)$, it is required to solve the perturbed Poisson equation

$\nabla^{2} \phi_{l}(r)=4 \pi G \rho_{l}(r)$, 
which is simply recovered from substituting the modal expansions of $\phi(r, \theta)$ and $\rho(r, \theta)$ in the Poisson equation. Explicitly the perturbed Poisson equation reads:

$\frac{1}{r} \frac{\mathrm{d}^{2}}{\mathrm{~d} r^{2}}\left(r \phi_{l}\right)-\frac{l(l+1)}{r^{2}} \phi_{l}-\frac{4 \pi G}{g_{0}} \frac{\mathrm{d} \rho_{0}}{\mathrm{~d} r} \phi_{l}=\frac{4 \pi G}{g_{0}} \frac{\mathrm{d} \rho_{0}}{\mathrm{~d} r} U_{l}$,

with boundary conditions

$\phi_{l}(0)=0 \quad$ and $\quad \frac{\mathrm{d}}{\mathrm{d} r} \phi_{l}(R)=\frac{(l+1)}{R} \phi_{l}(R)$

and $l \in\{0,2\}$ (Sweet 1950; Zahn 1966; Mathis \& Prat 2019, Appendix A).

Based on Lee \& Baraffe (1995) and Mathis \& Prat (2019), we now define the pseudo-radial coordinate $a$ as follows:

$r=a[1+\varepsilon(a, \theta)]$.

At this point, we perform a transformation from a spherical coordinate system $(r, \theta, \varphi)$ to a spheroidal coordinate system $(a, \theta, \varphi)$. $\varepsilon$ represents a dimensionless deformation factor, defined in the spheroidal coordinate system. Again following Mathis \& Prat (2019, Appendix A), an expression for the modal amplitudes of $\varepsilon$ can be derived:

$\varepsilon_{l}(a)=-\frac{\phi_{l}\left(A_{\mathrm{S}}\right)+U_{l}\left(A_{\mathrm{S}}\right)}{g_{0}\left(A_{\mathrm{S}}\right)} \frac{a^{3}}{A_{\mathrm{S}}^{4}}$,

with $A_{\mathrm{S}}$ the surface pseudo-radius of the deformed star. The coordinate mapping described by these expressions for $\varepsilon$ and $a$ is more accurate than the linear approximation used by Mathis \& Prat (2019) and closely agrees with the physical mapping described by, e.g., Zahn (1966) and Mathis \& Zahn (2004), so that $r$ is equal to the deformed surface radius $R_{\mathrm{S}}(\theta)$ at $a=A_{\mathrm{S}}$. However, while this physical mapping has a singularity at the stellar centre, our cubic expression ensures that $a \simeq r$ when $a \rightarrow 0$.

From the expressions for the modal amplitudes of the pressure, density and deformation factor (Eqs. 4, 5 \& 10), it can be seen that the centrifugal acceleration enters the expressions of the perturbed quantities in the form of a second order perturbation in the stellar rotation rate $\Omega$. In the expression for $\varepsilon_{l}(a)$ given in Eq.(10) we have a dominant contribution from $U_{l}$ (compared to $\left.\phi_{l}\right)$. Hence, $\varepsilon_{l=0}\left(\varepsilon_{l=2}\right)$ is positive (negative), and the deformation factor $\varepsilon$ is positive in the direction of the equator.

To facilitate the calculation of $\varepsilon_{l}$, we can map the radial coordinate $r_{0}$ and the surface radius $R$ of the spherically symmetric (non-deformed) stellar model onto $a$ and $A_{\mathrm{S}}$, respectively. Within their respective stellar models, both the $r_{0}$ and $a$ coordinates coincide with the radial isobaric coordinate. Thus, we get

$\varepsilon_{l}\left(r_{0}\right)=-\frac{\phi_{l}(R)+U_{l}(R)}{g_{0}(R)} \frac{r_{0}^{3}}{R^{4}}$.

\subsubsection{Generalised Laplace tidal equation}

The Laplace tidal equation is an eigenvalue equation that can be derived from the oscillation equations by using the TAR. Its simple form immediately demonstrates the simplifying power of the latter approximation. Following Lee \& Saio (1997), it can be written as:

$\mathcal{L}_{v m}^{\text {class. }}\left[\Theta_{v k m}\right]=-\Lambda_{v k m}^{\text {class. }} \Theta_{v k m}$, with the operator $\mathcal{L}_{v m}^{\text {class. }}$ defined as:

$$
\begin{aligned}
\mathcal{L}_{v m}^{\text {class. }}= & \frac{1-x^{2}}{1-v^{2} x^{2}} \partial_{x}^{2}-\frac{2 x\left(1-v^{2}\right)}{\left(1-v^{2} x^{2}\right)^{2}} \partial_{x} \\
& +\left[\frac{m v\left(1+v^{2} x^{2}\right)}{\left(1-v^{2} x^{2}\right)^{2}}-\frac{m^{2}}{\left(1-x^{2}\right)\left(1-v^{2} x^{2}\right)}\right] .
\end{aligned}
$$

In order to avoid confusion later on, we will refer to this equation as the classical Laplace tidal equation (abbreviated as CLTE). Here, $\partial_{x}=\partial / \partial x, x=\cos \theta$, and $v=2 \Omega / \omega$ the spin parameter. The latter is a measure of the effect of rotation; pulsation modes for which $v>1$ (resp. $v<1$ ) are known as sub- (resp. super-) inertial modes. The eigenfunctions $\Theta_{v k m}$ are the radial Hough functions, after S.S. Hough, who pioneered in solving the Laplace tidal equation (Hough 1898), and $\Lambda_{v k m}^{\text {class. }}$ represent the eigenvalues of the equation. These radial Hough functions give the co-latitudinal distribution of the radial displacement of the star caused by the pulsations and in the limit $v \rightarrow 0$ reduce to $C P_{l}^{m}(x)$, with $C$ a constant and $P_{l}^{m}(x)$ the associated Legendre polynomial of degree $l$ and order $m$. The eigenvalues reduce to $\ell(\ell+1)$ without rotation.

The convention is adopted in which, considering $v>0$, positive azimuthal orders $(m>0)$ denote prograde modes and $m<0$ retrograde modes. Since in general for each pair $(m, v)$ the CLTE yields an infinite set of solutions, the ordering number $k$ is introduced. Inertial waves, such as $r$ modes (which are normal modes of global Rossby waves influenced by buoyancy) (Saio et al. 2018), are not present in non-rotating stars and have $k<0$. For $k \geq 0$, the ordering number is related to the spherical degree $l$ and azimuthal order through $\ell=|m|+k$ (Mathis \& Prat 2019).

To include the effect of the centrifugal acceleration in the Laplace tidal equation, it is re-derived in the spheroidal coordinate system by Mathis \& Prat (2019). The transition from a spherical to spheroidal coordinate system involves the following basis transformation:

$$
\left\{\begin{array}{l}
\widetilde{\boldsymbol{e}}_{a}=\left(1+\varepsilon+a \partial_{a} \varepsilon\right) \widehat{\boldsymbol{e}}_{r} \\
\widetilde{\boldsymbol{e}}_{\theta}=\partial_{\theta} \varepsilon \widehat{\boldsymbol{e}}_{r}+(1+\varepsilon) \widehat{\boldsymbol{e}}_{\theta}, \\
\widetilde{\boldsymbol{e}}_{\varphi}=(1+\varepsilon) \widehat{\boldsymbol{e}}_{\varphi}
\end{array}\right.
$$

with $\varepsilon \equiv \varepsilon(a, \theta)=\sum_{l=0,2} \varepsilon_{l}(a) P_{\text {leg }, l}(x)$ the deformation factor introduced above. The resulting generalised Laplace tidal equation (GLTE) then is:

$$
\begin{aligned}
\mathcal{L}_{v m}\left[w_{v k m}\right]= & \left(\frac{1-x^{2}}{\mathcal{D}}\right) \partial_{x}^{2} w_{v k m} \\
& +\left[\frac{\left(1-x^{2}\right) \partial_{x} \mathcal{E}}{\mathcal{D}}+\partial_{x}\left(\frac{1-x^{2}}{\mathcal{D}}\right)\right] \partial_{x} w_{v k m} \\
& -\left[\frac{m^{2}}{\left(1-x^{2}\right) \mathcal{D}}-m v \frac{\mathrm{d}}{\mathrm{d} x}\left(\frac{x \mathcal{C}}{\mathcal{D}}\right)-m v \frac{x C}{\mathcal{D}} \partial_{x} \mathcal{E}\right] w_{v k m} \\
= & -\Lambda_{v k m}(a) w_{v k m},
\end{aligned}
$$

where

$$
\begin{aligned}
\mathcal{A}(a, \theta) & =1+2 \varepsilon, \quad \mathcal{B}(a, \theta)=\mathcal{A}+\tan \theta \partial_{\theta} \varepsilon \\
\mathcal{C}(a, \theta) & =\mathcal{B} / \mathcal{A}, \quad \mathcal{D}(a, \theta)=\mathcal{A}\left[1-v^{2} \cos ^{2} \theta C^{2}\right] \\
\mathcal{E}(a, \theta) & =3 \varepsilon+a \partial_{a} \varepsilon .
\end{aligned}
$$

Utilising the same nomenclature as Mathis \& Prat (2019), the eigenfunction $w_{v k m}=w_{v k m}(a, \theta)$ is called the modified radial 
Hough function. Note that the eigenvalue $\Lambda_{v k m}(a)$ and modified radial Hough function have a parametric dependence on the pseudo-radial coordinate $a$, whereas in the spherically symmetric case there was no radial dependence of the solutions.

\subsubsection{Asymptotic frequencies}

Within the asymptotic regime, where the radial order $n$ of the modes is much larger than the spherical degree $\ell(n \gg \ell)$, and where we consider modes with $\omega \ll|N|$ and $\omega \ll\left|S_{\ell}\right|, N$ and $S_{\ell}$ being the Brunt-Väisälä and Lamb frequency respectively, asymptotic expressions for the pulsation frequencies can be derived (Shibahashi 1979; Tassoul 1980; Unno et al. 1989). Following Mathis (2009) and Bouabid et al. (2013), these asymptotic expressions for the pulsation frequencies can be re-derived to include the effect of the centrifugal force. Mathis \& Prat (2019) find the angular pulsation frequencies to be:

$\omega_{n k m}=\frac{\int_{a_{1}}^{a_{2}} \frac{\Lambda_{v k m}^{1 / 2}(a) \bar{N}(a)}{a} \mathrm{~d} a}{(n+1 / 2) \pi}$

and the pulsation periods:

$$
P_{n k m}=\frac{2 \pi^{2}(n+1 / 2)}{\int_{a_{1}}^{a_{2}} \frac{\Lambda_{v k m}^{1 / 2}(a) \bar{N}(a)}{a} \mathrm{~d} a} .
$$

Here, $\bar{N}(a)$ is the perturbed Brunt-Väisälä frequency profile (quantities denoted with a bar are defined within the spheroidal coordinate system). To account for the large diversity of pulsation mode cavities of $g$ modes with different identification $(k, m)$ or spin $v, \bar{N}^{2}(a)$ is calculated as a weighted average over the colatitude $\theta$ :

$\bar{N}^{2}(a)=\frac{\int_{0}^{\pi} H_{r}(a, \theta) N^{2}(a, \theta) \sin \theta \mathrm{d} \theta}{\int_{0}^{\pi} H_{r}(a, \theta) \sin \theta \mathrm{d} \theta}$,

where $H_{r}(a, \theta)$ is the radial eigenfunction that corresponds to the eigenvalue $\Lambda_{v k m}(a)$ in Eqs.(16) and (17), and

$N^{2}(a, \theta)=-\frac{\bar{g}}{r}\left[\frac{\mathrm{d} \ln \bar{\rho}}{\mathrm{d} \ln r}-\frac{1}{\bar{\Gamma}_{1}} \frac{\mathrm{d} \ln \bar{P}}{\mathrm{~d} \ln r}\right]$,

with $\bar{\Gamma}_{1}=(\partial \ln \bar{P} / \partial \ln \bar{\rho})_{\bar{S}}$ the perturbed adiabatic exponent defined at constant (perturbed) entropy $\bar{S}$. The different quantities on the right-hand side of Eq.(19) all depend on both $a$ and $\theta$. In the integral in Eq. (17), $a_{1}$ and $a_{2}$ are the inner- and outer-boundaries of the mode cavity, determined as the region(s) within the star where $\bar{N}^{2}(a)>0$.

Another result from asymptotic theory is that, for a nonrotating, non-magnetic g-mode pulsator without chemical gradients, the pulsation periods of modes with consecutive radial orders are equidistant. The differences in mode periods, $\Delta P_{n k m}=$ $P_{n+1, k m}-P_{n k m}$ thus remain constant when plotted as a function of the period $P_{n k m}$ or radial order $n$ (Miglio et al. 2008). When constructing these so-called period spacing patterns for more realistic stellar models (including rotation and chemical gradients), they will deviate from a constant, as first observed for a mainsequence massive early-type star by the CoRoT satellite (Degroote et al. 2010). Comparing theoretical and observed period spacing patterns then allows us to deduce information about rotation and chemical mixing in the deep interior of stars (Aerts 2021).

\subsection{Computational implementation}

The computational method to derive the asymptotic GIW frequencies from the solutions to Eq. (16) is provided in Appendix B. The implementation of the analytical framework of Mathis \& Prat (2019) described above consists of three parts. First, a non-rotating 1D stellar structure model is calculated and perturbed for a selected uniform rotation rate, following Sect. 2.1.1. These 1D, non-rotating stellar structure models are calculated using the code MESA ${ }^{1}$ (Modules for Experiments in Stellar Astrophysics; Paxton et al. 2019 and references therein). The resulting 2D (radial and latitudinal) profiles for $r(a, \theta)$ and $\varepsilon$ are subsequently used as input for solving the GLTE given in Sect. 2.1.2. The solutions of the GLTE then allow us to compute the asymptotic pulsation frequencies based on Eq. (16).

The computational implementation of the first step involves solving the perturbed Poisson equation (e.g. through an iterative shooting scheme) for $l=0$ and $l=2$, yielding the modal amplitudes $\phi_{l}(r)$. These, in turn, are used to calculate the modal amplitudes of the pressure and density (Eqs. $4 \& 5$ ). Two challenges occur in the computation of the perturbed Brunt-Väisälä frequency profile:

i The calculation of $\bar{\Gamma}_{1}$ (at constant entropy $\bar{S}$ ) requires detailed knowledge on how the equation of state changes under influence of the centrifugal deformation. This would require the framework of Mathis \& Prat (2019) to be directly implemented in MESA.

ii High levels of numerical noise are introduced by the numerical derivatives of the physical quantities (such as the pressure and density) with respect to $r$ and $a$, which is a well-known problem in stellar structure and evolution codes (Paxton et al. 2013).

The details of how we treated these aspects are described in Appendix A.

\subsubsection{Solver for the GLTE}

Several numerical methods are available for solving an eigenvalue problem such as the Laplace tidal equation, a number of which are discussed in Wang et al. (2016). As in Mathis \& Prat (2019), the Chebyshev collocation method (Boyd 1976) is chosen. In such a method, the eigenfunctions of the problem, in this case the modified radial Hough functions, are expanded on a basis of $N$ Chebyshev polynomials, where $N$ is the number of collocation points. For the current work, we improved an existing solver for the CLTE of Prat et al. (2019) and Van Beeck et al. (2020), which was developed by one of us (VP), with two modifications.

Contrary to the CLTE, the GLTE has a parametric dependence on the radial coordinate (in this case $a$ ). In other words, at each cell of the MESA model, the solutions of the GLTE will be different, since $\varepsilon(a, \theta)$ depends on $a$. Furthermore, in order to select the solution corresponding to the desired mode identification $(k, m)$ among the set of $N$ solutions, the solver requires an estimate for the eigenvalue $\Lambda_{v k m}(a)$. At the centre of the model, where $\varepsilon=0$, the eigenvalue can be estimated by the solutions of the CLTE tabulated in the TAR module of the stellar pulsation code GYRE (Townsend \& Teitler 2013; Townsend et al. 2018). Going from the centre to the surface, at each subsequent cell, the estimate is then provided by the eigenvalue from the previous cell. Since the effect of the centrifugal force increases from

1 MESA version r11701. For more information about the different versions, see http://mesa. sourceforge.net/. 
the centre to the surface (see Eqs. $2 \& 10$ ) these eigenvalues will increasingly diverge from the value at the centre. To keep the computation time manageable, an appropriate number of sample points in which the GLTE is solved, must be selected. Thanks to the smooth behaviour of $\Lambda_{v k m}(a)$ (see Sect.3) it suffices to calculate solutions for $\sim 10$ cells. These points are chosen equidistant in cell index in the MESA model rather than in physical distance. In that way, regions with higher cell density get a higher sampling. $\Lambda_{v k m}(a)$ profiles, required for asymptotic frequency calculations (see Eq. 16) are retrieved through quadratic interpolation.

The calculation of the coefficients $\mathcal{A}, \mathcal{B}, \mathcal{C}, \mathcal{D}, \mathcal{E}$ and their derivatives, which make up the coefficients of the GLTE, is subject to discontinuities when done from numerical differentiation. We therefore relied on analytical differentiation where possible. Only for $\mathcal{E}$ and its derivative with respect to the co-latitudinal coordinate $x$ we approximate the term $a \partial_{a} \varepsilon$ by $3 \varepsilon$ in order to avoid numerical issues. This approximation is justified by the cubic polynomial behaviour of the deformation factor. The coefficients, as implemented in the solver for the GLTE, are:

$$
\begin{aligned}
\partial_{x} \varepsilon & =3 x \varepsilon_{l=2} \\
\mathcal{A} & =1+2 \varepsilon \\
\mathcal{B} & =\mathcal{A}-3 \varepsilon_{l=2}\left(1-x^{2}\right) \\
\mathcal{C} & =\mathcal{B} / \mathcal{A} \\
\mathcal{D} & =\mathcal{A}\left(1-v^{2} x^{2} C^{2}\right) \\
\mathcal{E} & \simeq 6 \varepsilon
\end{aligned}
$$

with $\varepsilon_{l=2}=\varepsilon_{l=2}(a)$ the modal amplitude in the expression for $\varepsilon=\varepsilon(a, \theta)=\sum_{l=0,2} \varepsilon_{l}(a) P_{\text {leg }, l}(x)$. The respective derivatives with respect to $x=\cos \theta$ are:

$$
\begin{aligned}
\partial_{x} \mathcal{A} & =6 x \varepsilon_{l=2} \\
\partial_{x} \mathcal{B} & =12 x \varepsilon_{l=2} \\
\partial_{x} C & =\frac{1}{\mathcal{A}^{2}}\left(\mathcal{A} \partial_{x} \mathcal{B}-\mathcal{B} \partial_{x} \mathcal{A}\right) \\
\partial_{x} \mathcal{D} & =\partial_{x} \mathcal{A}\left(1-v^{2} x^{2} C^{2}\right)-2 \mathcal{A C} x v^{2}\left(C+\partial_{x} C\right) \\
\partial_{x} \mathcal{E} & =18 x \varepsilon_{l=2} .
\end{aligned}
$$

\section{Numerical results}

\subsection{Covered parameter space of equilibrium models}

With the goal of exploring the effect of the centrifugal acceleration on the stellar structure and on high-order g-mode pulsations in more depth than was done in the proof-of-concept by Mathis \& Prat (2019), the methodology described in the previous section has been applied to a range of MESA equilibrium models with varying input parameters. The ranges and values for these parameters are shown in Fig. 1.

- mass: the masses of the MESA models are chosen within the joint mass range of $\gamma$ Dor $\left[1.4,1.9 \mathrm{M}_{\odot}\right.$ ] (Mombarg et al. 2019) and SPB [3, $9 \mathrm{M}_{\odot}$ ] (Pápics et al. 2017, Pedersen et al., submitted) stars. The physical conditions inside these types of pulsating stars allow the TAR to be applied and these are the two types of main-sequence stars for which g-mode period spacing patterns are observed.

- age: the age of the stellar models is quantified in terms of their core hydrogen-mass fraction $X_{\mathrm{c}}$. Each model has been computed with an initial hydrogen mass fraction $X_{\text {ini }}=0.715$ and evolved to its specified $X_{\mathrm{c}}$.

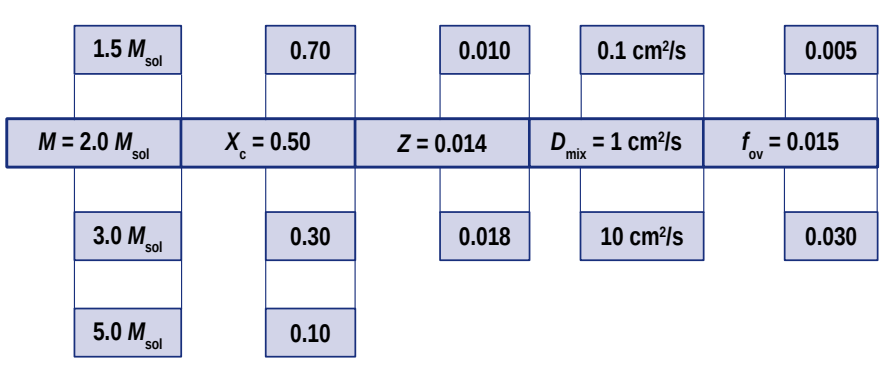

Fig. 1. Selected ranges and values for the input parameters of the 1D stellar structure models used in this work.

- metallicity: the range in initial metal mass fraction, $Z$, covers values typical for stars in our Milky Way in the considered mass range. By considering a range, we assess how the change in opacity caused by $Z$ affects the Brunt-Väisälä profile and the g-mode pulsation frequencies.

- envelope mixing: the amount of mixing that occurs in the radiative envelope of the star is quantified by the diffusion coefficient $D_{\text {mix }}$ and affects the profiles of the mass fractions, $X_{i}$, of all the isotopes considered in the chemical mixture adopted as input physics. As such, $D_{\text {mix }}$ has an influence on the Brunt-Väisälä frequency. In each of the calculated MESA models, we considered $D_{\text {mix }}$ to be constant throughout the radiative envelope, adopting values typical for g-mode pulsators (Van Reeth et al. 2016; Moravveji et al. 2016).

- convective core overshooting: this process is of major importance for stellar evolution in the considered mass range, yet it is least known among the various ingredients to be chosen as input physics (Claret \& Torres 2019; Johnston et al. 2019; Li et al. 2019a; Tkachenko et al. 2020). Different formalisms exist to describe this overshoot region. We adopted a diffusive exponentially-decaying overshooting (Freytag et al. 1996; Herwig 2000) quantified by the overshooting parameter $f_{\mathrm{ov}}$.

Furthermore, each model is computed using the AGSS09 chemical abundances derived by Asplund et al. (2009) and a mixing length $\alpha_{\mathrm{MLT}}=2$ within the mixing length theory developed by Henyey et al. (1965). For convergence purposes, a hot wind with Vink scaling factor of 1 is turned on Vink et al. (2001). In order to avoid numerical issues, rotation is not taken into account during the MESA model calculations. The effects of rotation enter in our models by applying the perturbation method described in Sect. 2.1.1.

\subsection{Solutions of the GLTE}

Here, we investigate the difference in solutions of the GLTE and CLTE. To illustrate these, we pick one baseline equilibrium model from our grid setup, as indicated in the highlighted row in Fig. 1. The behaviour is found to be equivalent for the other models so we do not discuss these results for brevity.

Fig. 2 shows the solution spectrum for the GLTE for $m=2$ with even and odd eigenfunctions. Four classes of solutions can be distinguished (Lee \& Saio 1997): (i) the prograde g modes with $v>0$ and positive eigenvalues $\Lambda_{v k m}(a)$, (ii) retrograde $g$ modes with $v<0$ and positive eigenvalues, (iii) Rossby modes which are retrograde and have negative eigenvalues, and (iv) prograde convective modes with negative eigenvalues. Just as Rossby modes, the latter only appear in rotating stars and only 


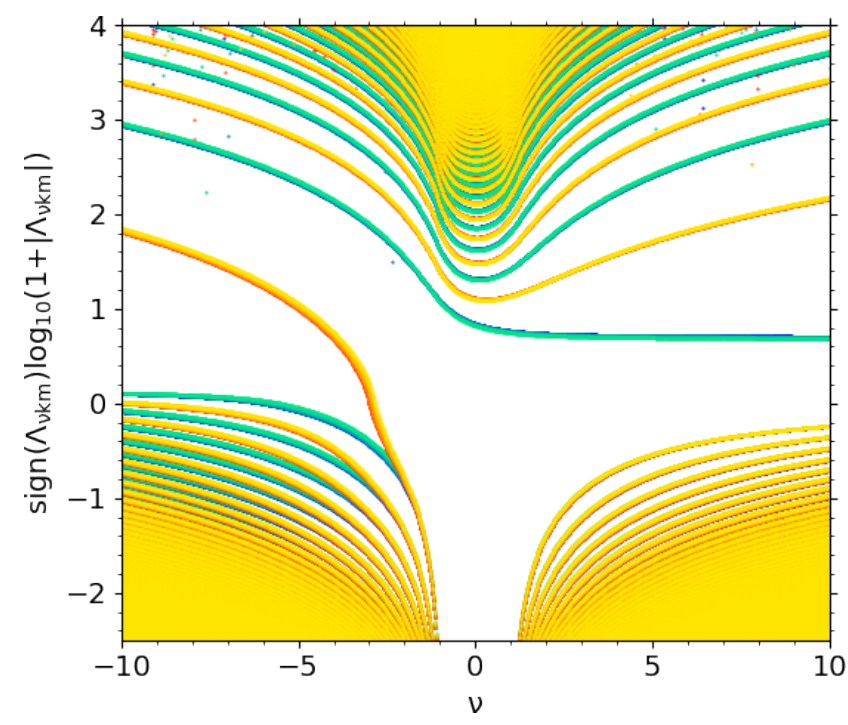

Fig. 2. Solution spectrum of the generalised Laplace tidal equation for modes with $m=2$. Modes with even eigenfunctions are shown in blue, those with odd eigenfunctions in orange. Light/dark colours correspond to solutions near the surface/core. The equilibrium model used as input for the computations has parameters as in the highlighted row in Fig. 1.

exist for $|v|>1$. Specifically, these modes are able to propagate in convective regions under the joint force of the Coriolis acceleration and buoyancy.

Keeping in mind that the solutions plotted in the darkest colours correspond to those for the core and are therefore equivalent to the solutions of the CLTE (see Sect. 2.2.1), Fig. 2 reveals that the centrifugal deformation of the star causes a gradual shift in the eigenvalues. Whether this is an upwards or downwards shift depends on the mode identification $(k, m)$ (we recall that solutions for negative spin parameters $v$ and $m>0$ are equivalent to solution with $v>0$ and $m<0$ ). The numerical 'noise' visible at higher values of $|v|$ are an artefact due to the limited number of Chebyshev collocation points $(N=200)$, but it can be seen that this does not affect the selected solutions. We obtained that an increase in the number of collocation points causes a decrease in this noise.

The (normalised) eigenfunctions $w_{v k m}$ of the GLTE for a ( $k=0, m=2$ ) mode with $v=2$ are shown in Fig. 3. Equivalent figures for $v=0.5$ (super-inertial) and $v=9$ (sub-inertial) are displayed in Fig. 4. The eigenfunctions of the GLTE differ increasingly from those of the CLTE as the distance from the centre of the model to the surface increases. More specifically, the eigenfunctions migrate inwards, towards the equator $(x=0)$, causing a narrowing of the overall shape of the eigenfunctions. Similar behaviour is observed for other modes, such as prograde dipole $(k=0, m=1)$ modes shown in Fig. 5, retrograde quadrupole $(k=0, m=-2)$, retrograde Rossby $(k=-2, m=-1)$, quadrupole zonal $(k=2, m=0)$, and $(k=1, m=1)$ oscillation modes. Off-equator extrema, such as for the eigenfunctions of $(k=0, m=-2)$ and $(k=-2, m=-1)$ modes shown in Fig. 5, experience net inward shifts towards the equator.

Fig. 6 shows the eigenfunctions at the surface $\left(a=A_{\mathrm{S}}\right)$ and the $\Lambda_{v k m}(a)$ profiles for a $M=1.5 \mathrm{M}_{\odot}, X_{\mathrm{c}}=0.50\left(X_{\mathrm{c}} / X_{\mathrm{ini}}=\right.$ 0.70 ) model for seven different rotation rates ranging from $\Omega=$ $0.1 \Omega_{\mathrm{c}}$ to $\Omega=0.7 \Omega_{\mathrm{c}}$. The figure is again for a quadrupole sectoral $(k=0, m=2)$ mode. We find that the narrowing of the

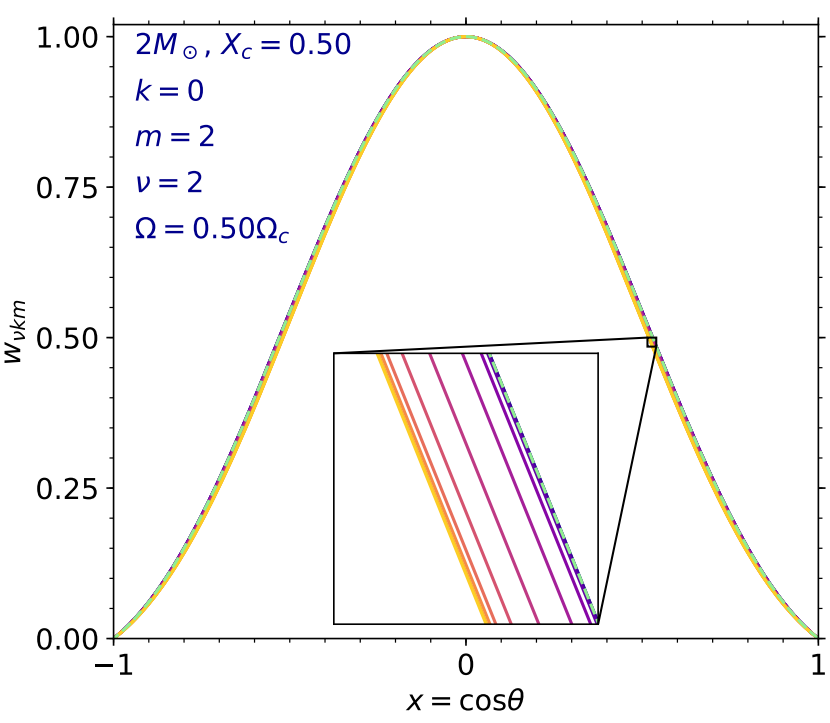

Fig. 3. Modified radial Hough functions $w_{v k m}(a, \theta)$ (normalised) for $v=$ 2 and $\Omega / \Omega_{\mathrm{c}}=0.50$. Solutions are plotted in a colour range from indigo to yellow from the stellar core to the surface. The green dashed line shows the solution of the CLTE. The number of collocation points for this computation was $N=200$.

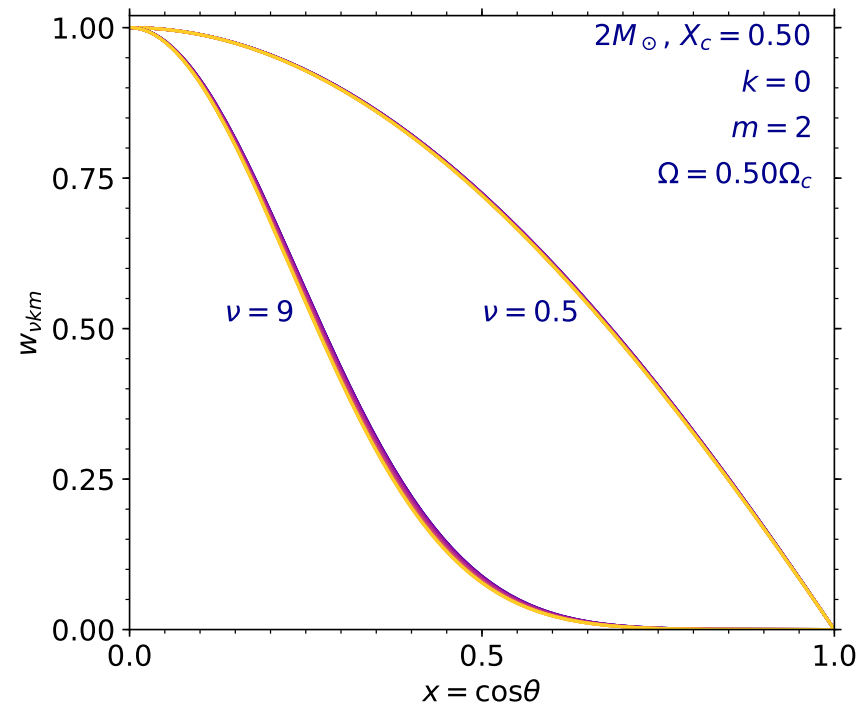

Fig. 4. Modified radial Hough functions $w_{v k m}(a, \theta)$ (normalised) for $\Omega / \Omega_{\mathrm{c}}=0.50$ and two different spin parameters. The $2.0 \mathrm{M}_{\odot}, X_{\mathrm{c}}=0.50$ equilibrium model was used as input. The colour scheme and number of collocation points are identical to that of Fig. 3 (CLTE solutions are left out for clarity).

shape of the eigenfunctions and the divergence of the eigenvalues from the value at the centre increase with increasing rotation rate, as expected.

\subsection{Asymptotic period spacing patterns}

We solved the GLTE for each model in our grid, covering a range of spin parameters for each of the models (see Appendix B). We restricted to three specific mode identifications: prograde dipole sectoral $(k=0, m=1)$ modes, prograde quadrupole sectoral $(k=0, m=2)$ modes, and retrograde Rossby modes with $(k=-2, m=-1)$. The reason for this choice is that these GIWs 

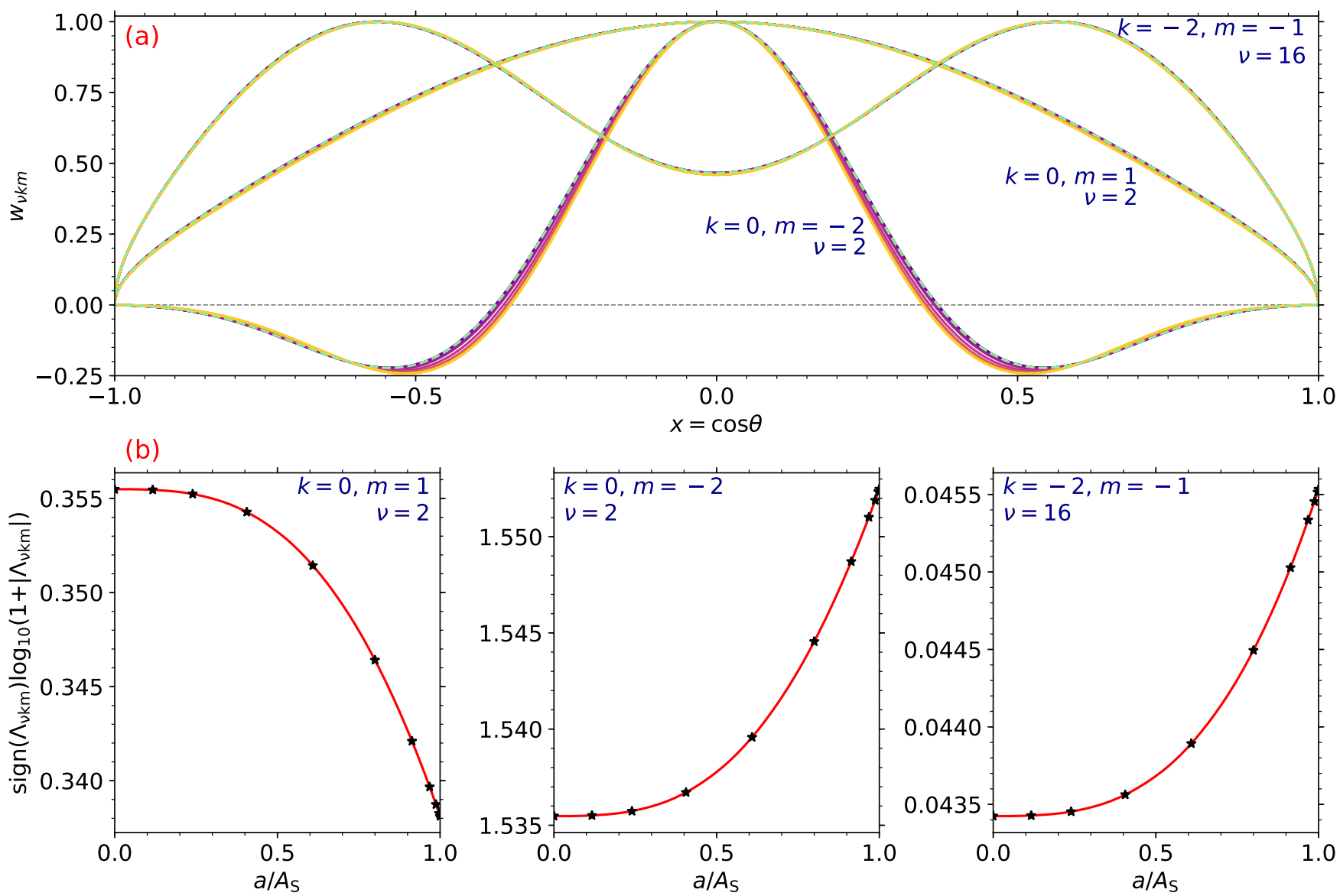

Fig. 5. (a) Modified radial Hough functions $w_{v k m}(a, \theta)$ (normalised) for three different modes (with specified spin parameter $v$ ). Colour conventions are the same as those in Fig. 3. (b) Eigenvalue $\Lambda_{v k m}(a)$ profiles corresponding to the eigenfunctions in $(a)$. Points indicated with a $\star$ correspond to eigenvalues obtained by solving the GLTE. Red points are found via quadratic interpolation. The considered number of collocation points was $N=200$.

are most often observed in rotating g-mode pulsators (Pápics et al. 2017; Li et al. 2020). The computed period spacing pattern for prograde dipole modes of the baseline model for $X_{\mathrm{c}}=0.50$ $\left(X_{\mathrm{c}} / X_{\mathrm{ini}}=0.70\right)$ is shown in Fig. 7. By comparing the centrifugally deformed period spacing pattern with their spherically symmetric counterpart, we find that the spacing values increase under the influence of the centrifugal acceleration. This increase is largest at lowest radial orders (short pulsation periods). Similar behaviour is found for $(k=0, m=2)$ modes.

Essentially two effects are at play here: on the one hand, the centrifugal acceleration directly affects the g-mode pulsations, via a $\Lambda_{v k m}(a)$-profile that changes throughout the star from $a=$ 0 to $a=A_{\mathrm{S}}$ instead of just one $\Lambda_{v k m}^{\text {class. }}$ value for $r_{0}=0 \rightarrow r_{0}=R$. On the other hand, the stellar shape, including the Brunt-Väisälä frequency profile and the volume, are perturbed, which has an indirect on the $g$-mode pulsations. In order to isolate the effect of this deformation, we re-calculated the period spacing pattern for the centrifugally deformed star for $\Lambda_{v k m}(a)=\Lambda_{v k m}^{\text {class. }}$, with $\Lambda_{v k m}^{\text {class. }}$ the eigenvalue of the CLTE. This period spacing pattern is plotted in red in Fig. 7. Similarly, the purple period spacing pattern was calculated by setting $\bar{N}(a)=N_{0}\left(r_{0}\right)$ and $a=r_{0}$, isolating the effect of the $a$-dependence of the solutions of the GLTE. Both effects lead to a net increase in the period spacings. Also for the $(k=-2, m=-1)$ Rossby modes shown in Fig. 8, a net upward shift of the period spacing pattern occurs. The effect is larger at longer pulsation periods, which for Rossby modes is equivalent to low radial orders (Saio et al. 2018). In some cases, such as for retrograde $\mathrm{g}$ modes with $(k, m)=(0,-1)$, we do find a net decrease in the period spacings $\Delta P_{n k m}$. In such cases the $\Lambda_{v k m}(a)$-value increases under the influence of the centrifugal acceleration, and counteracts the effect of the centrifugally modulated Brunt-Väisälä frequency profile $\bar{N}(a)$.

\subsection{Detectability in space-based photometric observations}

To quantify the effect the centrifugal deformation of the star on the pulsation frequencies, we computed the frequency differences between asymptotic frequencies calculated in the classical formulation of the TAR, and those calculated in the generalised formulation (through Eq. 16). We consider these frequency differences as a function of the radial order $n$, as this is similar to the common diagnostic observables used in g-mode asteroseismic modelling (Aerts et al. 2018). We compare the obtained frequency differences with the frequency resolutions $\left(1 / T_{\mathrm{obs}}\right)$ of Kepler and TESS light curves covering quasi-continuously observation times of $T_{\mathrm{obs}}=4$ years and $T_{\mathrm{obs}}=351$ days, respectively. In this way, we were able to deduce the radial orders $n_{\max }$ for which the frequency differences are expected to be detectable in the absence of instrumental effects and assuming excellent knowledge of the equilibrium models representing an observed star. In reality asteroseismic modelling never represents the stellar oscillations perfectly, meaning that the optimal reported $n_{\max }$ 

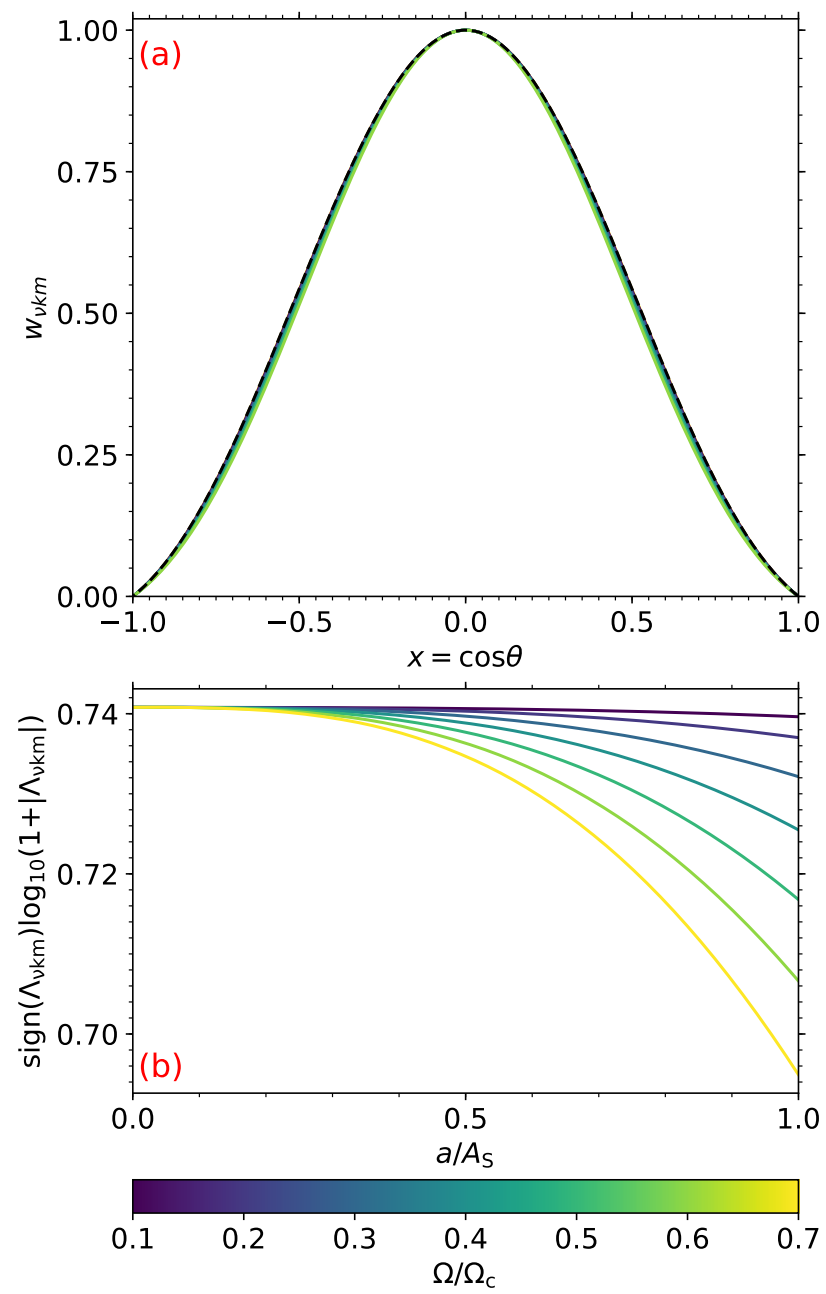

Fig. 6. (a) Modified radial Hough functions $w_{v k m}\left(a=A_{\mathrm{S}}, \theta\right)$ for seven different rotation rates and $v=2$. (b) Eigenvalue $\Lambda_{v k m}(a)$ profiles corresponding to the eigenfunctions in $(a)$.

leading to the largest frequency differences due to the centrifugal deformation are in fact lower limits of detectability in real applications of asteroseismology. The results for prograde dipole $(k=0, m=1)$ modes in the central $\left(2.0 \mathrm{M}_{\odot}, X_{\mathrm{c}}=0.50\right)$ model rotating at $0.15 \Omega_{\mathrm{c}}$ are displayed in Fig. 9. These computations were done for all the models in our grid and the considered modes. The results are listed in Table 1.

To assess whether the centrifugal acceleration is a necessary ingredient in asteroseismic modelling, we computed the fractional differences between asymptotic frequencies in centrifugally deformed and spherically symmetric stars for a range of rotation rates $\Omega / \Omega_{\mathrm{c}} \in[0.10-0.70]$. For these calculations, the central $2 \mathrm{M}_{\odot}, X_{\mathrm{c}}=0.5$ equilibrium model was used. We restricted these tests to prograde dipole sectoral $(k=0, m=1)$ modes, as these are most frequently observed. The results are displayed in Fig. 10. The middle and bottom panel of this figure show that the deformation of the stellar structure and the divergence of the eigenvalues of the GLTE each cause an increase of $\delta f_{n k m} / f_{n k m}$ (in absolute value) for increasing $\Omega=0.1 \Omega_{\mathrm{c}}$ to $0.7 \Omega_{\mathrm{c}}$. The fractional frequency differences caused by both effects are on the order of $1 \%$. And while the indirect effect of the deformed stellar structure has a slightly larger impact, this again shows that both the deformation of the stellar structure itself and the generalisation of the TAR are required to accurately describe the effect of the centrifugal acceleration on GIW.

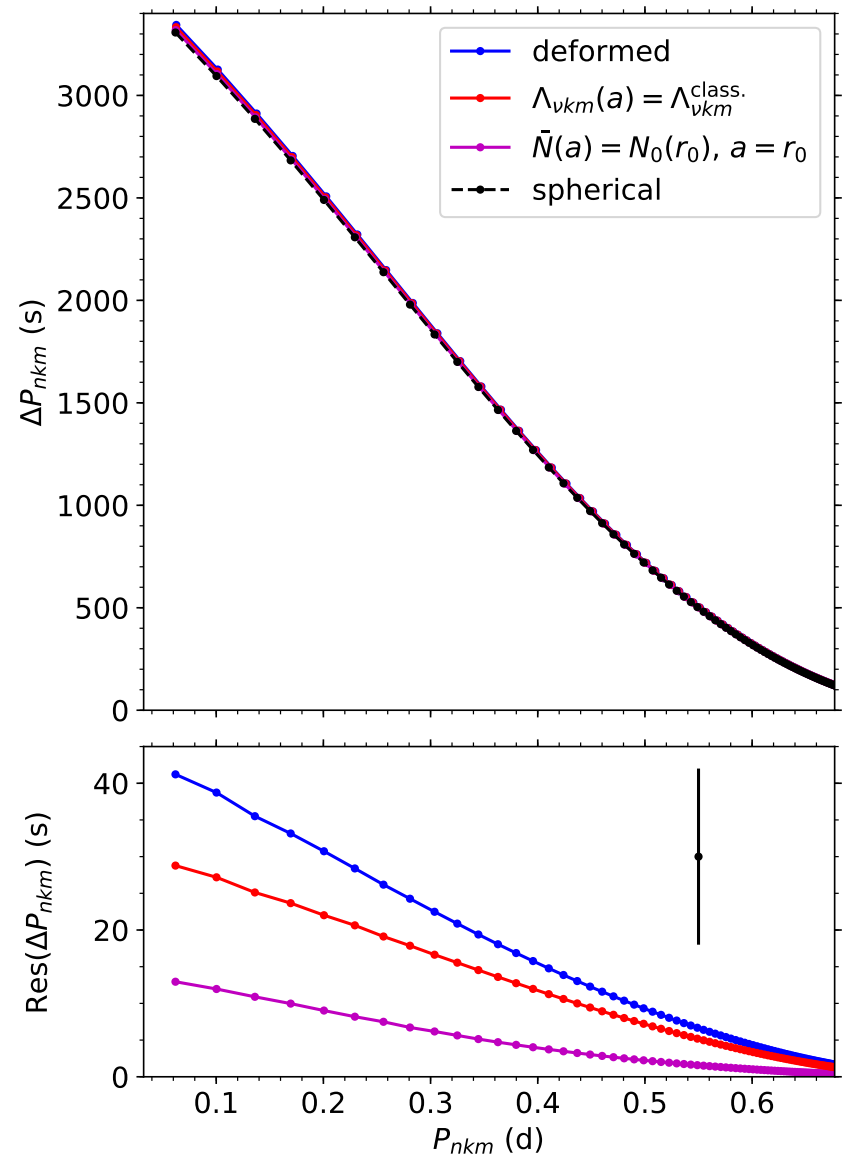

Fig. 7. Period spacing pattern in an inertial frame computed for $(k=$ $0, m=1)$ modes in a centrifugally deformed $2.0 \mathrm{M}_{\odot}, X_{\mathrm{c}}=0.50$ $\left(X_{\mathrm{c}} / X_{\text {ini }}=0.70\right), Z=0.014, D_{\text {mix }}=1 \mathrm{~cm}^{2} \mathrm{~s}^{-1}, f_{\text {ov }}=0.015$ equilibrium model at $\Omega / \Omega_{\mathrm{c}}=0.50$ (blue line). The black-dashed line shows the equivalent period spacing pattern for the same model, but without the effect of the centrifugal acceleration. The red and purple lines show the results for $N_{0}(r) \rightarrow \bar{N}(a) ; r \rightarrow a$ and $\Lambda_{v k m}^{\text {class. }} \rightarrow \Lambda_{v k m}(a)$, respectively. The bottom panel shows the differences with respect to the spherically symmetric period spacing pattern. The indicated global errors for the periods and period spacings are averages calculated from the uncertainties of $P$ and $\Delta P$ for a sample of $40 \gamma$ Dor stars by Van Reeth et al. (2015), where the horizontal bar is hardly visible as it represents $10^{-4} \mathrm{~d}$.

The frequency differences provide an assessment for the applicability of the analytical framework in Mathis \& Prat (2019) in terms of the fraction of the critical rotation rate $\Omega_{\mathrm{c}}$. For rotation rates near the critical value, i.e. $\Omega / \Omega_{\mathrm{c}}=0.80-0.99$ (not shown in Fig. 10) the behaviour of $\delta f_{n k m} / f_{n k m}$ deviates from the smooth curves in the figure for the lower values. This indicates that the assumptions in the analytical frame work (e.g., only taking $l=0$ and $l=2$ projections into account) no longer hold for higher rotation rates than those shown in Fig. 10. The case where the centrifugal acceleration is treated in a non-perturbative manner will be part of forthcoming work (Dhouib et al., in prep.).

\section{Discussion \& conclusions}

The results in the previous section reveal that the centrifugal deformation of the star implies a shift in the extrema of the eigenfunctions towards the equator of the star. This effect becomes more outspoken towards the surface, since the centrifugal acceleration (and hence the dimensionless deformation factor $\varepsilon$ ) becomes more important as one moves from the centre to the outer 


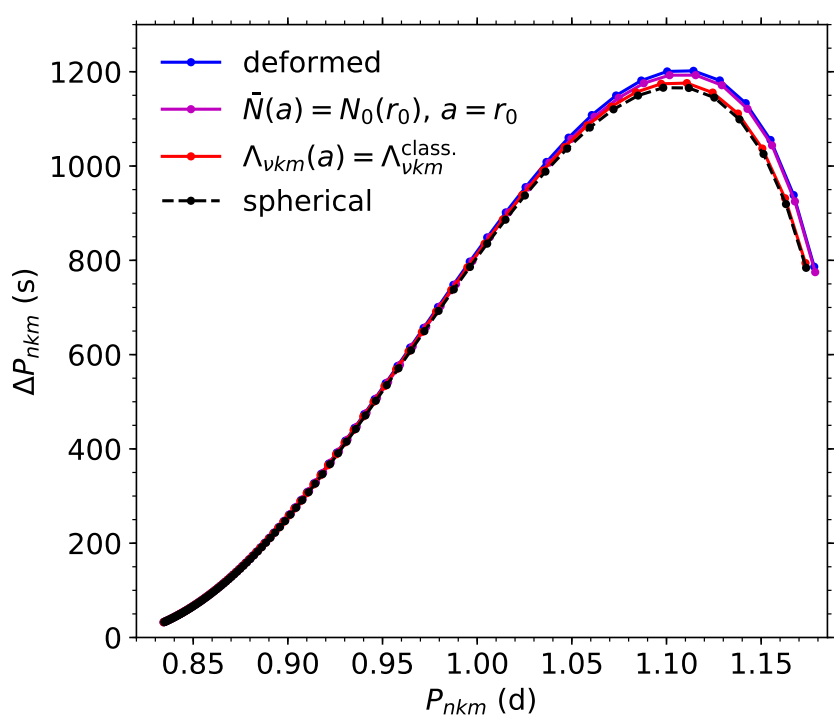

Fig. 8. Theoretical period spacing pattern in the inertial frame for ( $k=-2, m=-1)$ modes in the same equilibrium model as in Fig. 7 at $\Omega / \Omega_{\mathrm{c}}=0.50$ (blue line). The black-dashed line shows the equivalent period spacing pattern for the same model, but without the effect of the centrifugal acceleration. The red and purple show the isolated effect of $N_{0}(r) \rightarrow \bar{N}(a) ; r \rightarrow a$ and $\Lambda_{v k m}^{\text {class. }} \rightarrow \Lambda_{v k m}(a)$, respectively.

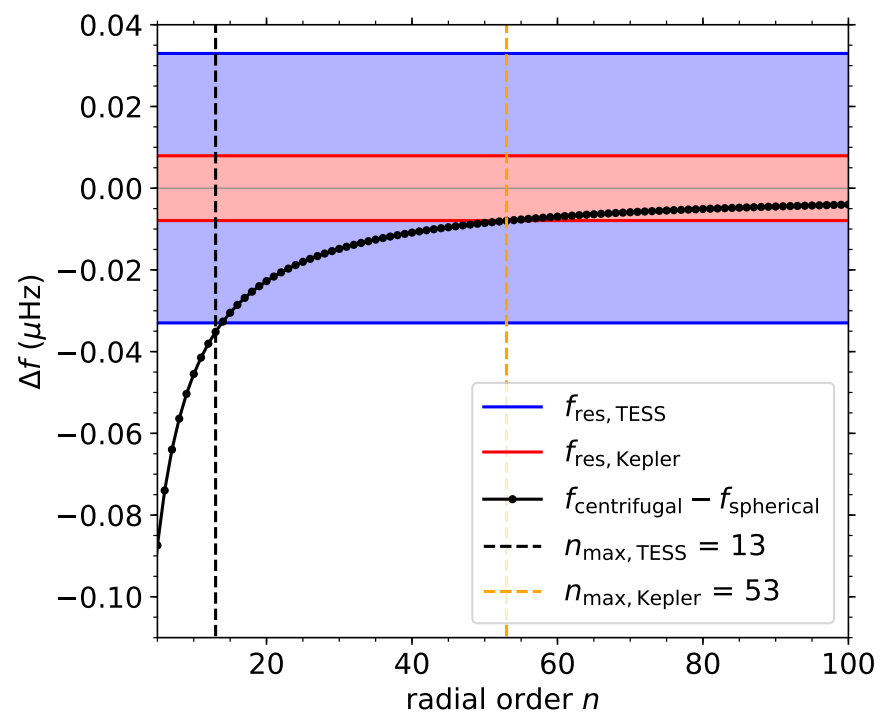

Fig. 9. Asymptotic frequency differences (black) for dipole $(k=0, m=$ 1) modes at $\Omega=0.15 \Omega_{\mathrm{c}}$ for the central $2.0 \mathrm{M}_{\odot}, X_{\mathrm{c}}=0.50 \mathrm{MESA}$ model. Red and blue bands represent the frequency resolution of Kepler and TESS respectively. The respective values of $n_{\max }$ are indicated by vertical dashed lines.

layers. The eigenfunctions centred around the equator reveal narrower maxima, while the extrema of the off-equator eigenfunctions move inwards, towards the equator. We thus find that the effect of the centrifugal acceleration modifies the one of the Coriolis acceleration included in the CLTE. More precisely, the Coriolis acceleration is responsible for a concentration of GIW towards the equator, while the centrifugal acceleration causes these equatorial bands to become narrower and the off-equator extrema of eigenfunctions shift inwards.

We found differences between the proof-of-concept study by Mathis \& Prat (2019) and the quantitative numerical results in
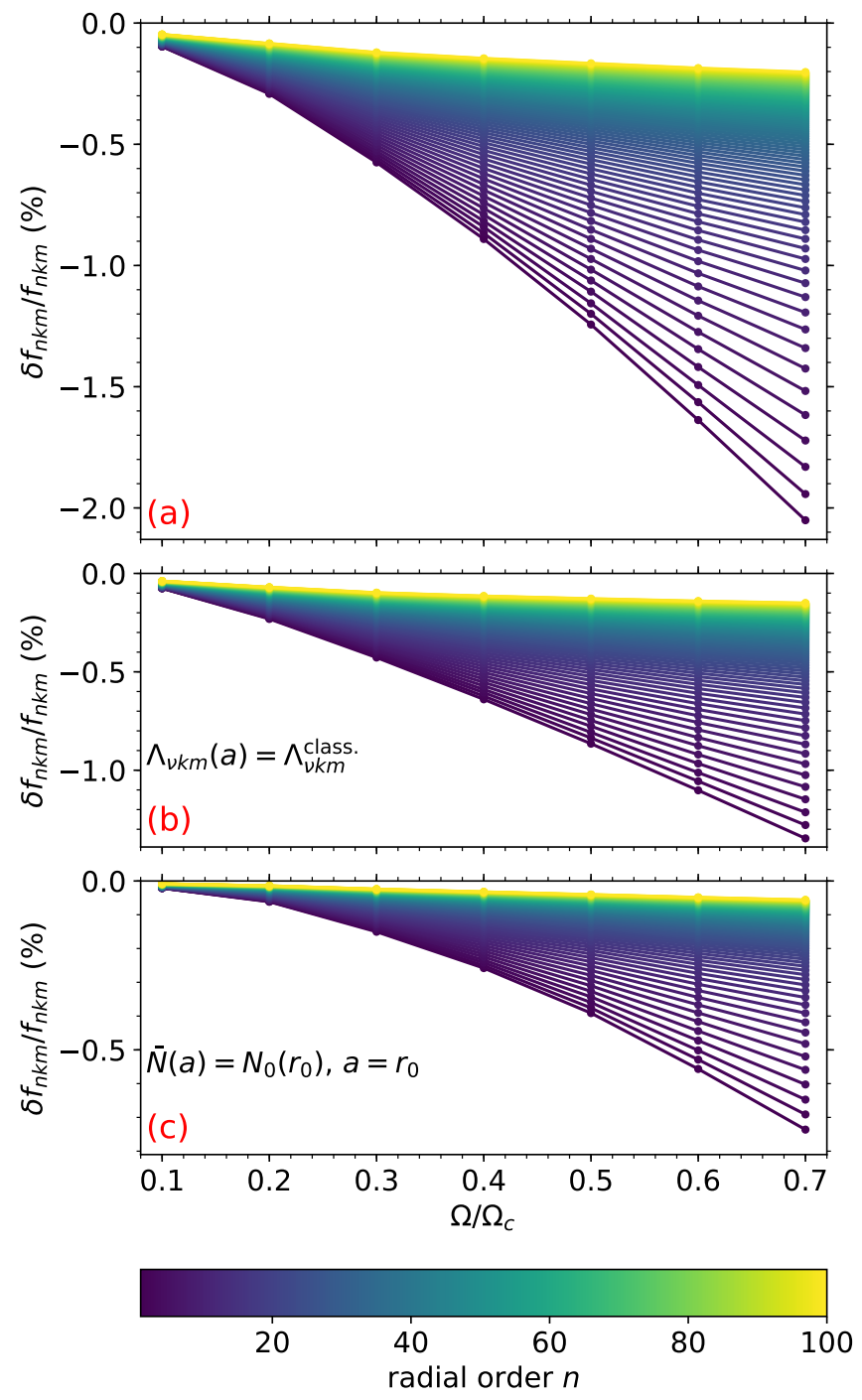

Fig. 10. (a) Fractional frequency difference $\delta f_{n k m} / f_{n k m}$, with $\delta f_{n k m}=$ $f_{\text {centrifugal }}-f_{\text {spherical }}$ and $f_{n k m}=f_{\text {centrifugal }}$ in function of the fractional rotation rate, for $n=1$ to $n=100$. Calculations based on central $2 \mathrm{M}_{\odot}$, $X_{\mathrm{c}}=0.50$ MESA model with $\Omega / \Omega_{\mathrm{c}} \in[0.10-0.70]$, exhibiting prograde dipole sectoral $(k=0, m=1)$ modes. (b) Same as (a), but now with the isolated effect of the deformation of the Brunt-Väisälä frequency profile and radial coordinate (achieved by setting $\Lambda_{v k m}(a)=\Lambda_{v k m}^{\text {class. }}$ ). (c) Same as (a), but now with the isolated effect of the deviation of the eigenvalue of the GLTE (achieved by setting $\bar{N}(a)=N_{0}\left(r_{0}\right)$ and $\left.a=r_{0}\right)$.

this work. The current new implementation uses a different approach for the computational aspects to solve the GLTE. In order to avoid numerical issues, Mathis \& Prat (2019) made use of linearized expressions for the coefficients of the GLTE. However, such an approach revealed the solutions to be rather dependent on the used number of collocation points. For this reason, we circumvented the use of numerical derivatives to solve the GLTE analytically where possible, as described in Sect. 2.2.1. In this way, the avoided crossings in the solution spectrum for the GLTE (Fig. 2; their Figs. 5\&6) are circumvented here. Moreover, the current numerical approach leads to relatively modest shift of the eigenfunctions towards the equator as shown graphically in our Figs. 3, 5\& 6, as an improvement compared to the more pronounced behaviour found in Figs. $7 \& 8$ in Mathis \& Prat (2019). 
Table 1. Potential detectability of the effect of the centrifugal acceleration in space-based photometric observations.

\begin{tabular}{|c|c|c|c|}
\hline model & $\begin{array}{c}f_{\text {rot }} \\
{[\mu \mathrm{Hz}]}\end{array}$ & $\begin{array}{c}n_{\max } \\
\text { (Kepler) }\end{array}$ & $\begin{array}{c}n_{\max } \\
\text { (TESS) }\end{array}$ \\
\hline $\begin{array}{l}\text { central model } \\
2.0 \mathrm{M}_{\odot}, X_{\mathrm{c}}=0.5, Z=0.014, \\
D_{\text {mix }}=1 \mathrm{~cm}^{2} / \mathrm{s}, f_{\mathrm{ov}}=0.015, \\
0.15 \Omega_{\mathrm{c}},(k, m)=(0,1)\end{array}$ & 4.35 & 53 & 13 \\
\hline $1.5 \mathrm{M}_{\odot}$ & 4.87 & 42 & 10 \\
\hline $3.0 \mathrm{M}_{\odot}$ & 3.72 & 62 & 16 \\
\hline $5.0 \mathrm{M}_{\odot}$ & 3.11 & 69 & 18 \\
\hline$X_{\mathrm{c}}=0.7$ & 6.01 & 74 & 19 \\
\hline$X_{\mathrm{c}}=0.3$ & 2.99 & 39 & 9 \\
\hline$X_{\mathrm{c}}=0.1$ & 1.88 & 28 & 6 \\
\hline$Z=0.010$ & 4.62 & 60 & 15 \\
\hline$Z=0.018$ & 4.14 & 50 & 13 \\
\hline$D_{\text {mix }}=0.1 \mathrm{~cm}^{2} / \mathrm{s}$ & 4.35 & 53 & 13 \\
\hline$D_{\operatorname{mix}}=10 \mathrm{~cm}^{2} / \mathrm{s}$ & 4.35 & 53 & 13 \\
\hline$f_{\mathrm{ov}}=0.005$ & 4.51 & 55 & 14 \\
\hline$f_{\mathrm{ov}}=0.030$ & 4.13 & 50 & 13 \\
\hline $0.10 \Omega_{\mathrm{c}}$ & 2.90 & 31 & 7 \\
\hline $0.20 \Omega_{\mathrm{c}}$ & 5.80 & 82 & 22 \\
\hline $0.30 \Omega_{\mathrm{c}}$ & 8.70 & $>100$ & 39 \\
\hline $0.40 \Omega_{\mathrm{c}}$ & 11.59 & $>100$ & 59 \\
\hline $0.50 \Omega_{\mathrm{c}}$ & 14.49 & $>100$ & 82 \\
\hline $0.60 \Omega_{\mathrm{c}}$ & 17.40 & $>100$ & $>100$ \\
\hline$k=0, m=2$ & 4.35 & $>100$ & 25 \\
\hline$(k, m)=(-2,-1), 0.20 \Omega_{\mathrm{c}}$ & 5.80 & $\ldots$ & 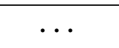 \\
\hline$(k, m)=(-2,-1), 0.30 \Omega_{\mathrm{c}}$ & 8.70 & 10 & $\ldots$ \\
\hline$(k, m)=(-2,-1), 0.40 \Omega_{\mathrm{c}}$ & 11.59 & 14 & $\ldots$ \\
\hline$(k, m)=(-2,-1), 0.50 \Omega_{\mathrm{c}}$ & 14.49 & 85 & 5 \\
\hline$(k, m)=(-2,-1), 0.60 \Omega_{\mathrm{c}}$ & 17.39 & $>100$ & 8 \\
\hline$(k, m)=(-2,-1), 0.70 \Omega_{\mathrm{c}}$ & 20.29 & $>100$ & 9 \\
\hline
\end{tabular}

Notes. The first entry of the table (above the line) contains the result for the central model, of which the MESA input parameters, fractional rotation rate and mode identification are given. Subsequently, each of the indicated parameters/rotation rates/mode identifications is varied with respect to the central model. The influence of the rotation rate is evaluated again separately for $(k, m)=(-2,-1)$.

In essence, the centrifugal acceleration affects two aspects within the theoretical description of g-mode pulsators. On the one hand, the stellar structure as a whole becomes deformed, which naturally leads to changes in the Brunt-Väisälä frequency profile and therefore in the cavities of the gravity modes. This is an indirect effect. On the other hand, the g-mode oscillation equations, in the form of the Laplace tidal equations, are altered and get a radial dependence, propagating into deformed solutions compared to those of the CLTE. This is a direct effect. In the case of prograde sectoral modes, these aspects have similar effects on the mode frequencies, as demonstrated in Sect. 3.3.

The detectability of the effect of the centrifugal acceleration in space-based photometry decreases with increasing stellar age (decreasing $X_{\mathrm{c}}$ ), increasing metallicity $Z$ and increasing core overshooting $f_{\text {ov }}$. Further, as seen in in Table 1, the value of $n_{\max }$ increases with increasing stellar mass and increasing rotation rate, since the deformation factor and rotation rate scale as $\varepsilon \sim \Omega^{2}$.

Comparing the $n_{\max }$ values with radial-order distributions of observed GIWs (Li et al. 2020, and Pedersen et al., submitted), we conclude that the values we obtained here correspond well with the observations. This implies that it should be possible to detect differences between theoretically computed pulsation frequencies assuming spherically symmetric versus deformed stellar models as computed in this work for real stars, provided that the comparison is done for models with the same input physics and rotation rate. The frequency differences as shown in Fig. 9 are of similar order as typical uncertainties of the observed frequencies used in forward asteroseismic modelling (Aerts et al. 2018).

Frequency differences caused by other approximations that are commonly made in the TAR, such as the Cowling approximation and the neglect of the horizontal component of the rotation vector, are smaller than or comparable to those caused by the centrifugal acceleration. This is shown in Appendix C, where we obtain relative differences $\lesssim 1 \%$ dependent on the stellar rotation rate, comparable to the differences between the TAR and the Mathis \& Prat (2019) framework. A comparison between the TAR and full 2D-computations carried out with ACOR by Ouazzani et al. (2017, Figure 1), gave similar results. This indicates that, just like the neglect of the centrifugal acceleration, other assumptions made within the TAR also have negligible or minor effects on the pulsation calculations. A limitation of the asymptotic expressions that is still present in the Mathis \& Prat (2019) framework, is that the effect of the centrifugal acceleration increases with decreasing radial order of the $g$ modes. At low radial order, both the TAR and the asymptotic expressions cease to be valid.

In practice, Fig. 10 reveals that the fractional frequency differences due to the centrifugal acceleration remain well below $1 \%$ for high-order $g$ modes in the asymptotic regime. Comparing this with typical fractional frequency differences in Aerts et al. (2018, Table 2), points out that the effect of the centrifugal acceleration is negligible compared to that introduced by some aspects of missing input physics, such as atomic diffusion in slow rotators among the g-mode pulsators (Mombarg et al. 2020) or near-core boundary mixing in fast rotating single SPB stars (Moravveji et al. 2016; Szewczuk \& Daszyńska-Daszkiewicz 2018, and Pedersen et al., submitted) and SPB binaries (Johnston et al. 2019). Hence, although the frequency differences induced by the centrifugal acceleration are expected to be detectable, one can ignore them for initial attempts of asteroseismic modelling. Once appropriate equilibrium models are found that explain well the overall structure in the measured period spacing pattern of a star, it is meaningful to test the effect of the centrifugal acceleration for those models to see if it brings an improved fit, particularly for stars with relatively fast rotation.

Following the conclusions of Mathis \& Prat (2019) and ours, a next logical step would be to include the effect of the centrifugal acceleration in stellar pulsation codes such as GYRE. Although our work has shown that the centrifugal acceleration can be treated as a lower-priority ingredient for forward asteroseismic modelling compared to other missing ingredients in the input physics, its effect is in principle detectable at the level of the mode computations. Hence, including it would lead to overall more realistic stellar pulsation predictions.

Acknowledgements. We thank the referee for their encouragement. We thank the MESA and GYRE developers for their efforts and for making their codes publicly available. The research leading to these results has received funding from the European Research Council (ERC) under the European Union's Horizon 2020 research and innovation programme (grant agreements $\mathrm{N}^{\circ} 670519$ : MAMSIE with PI Aerts and $N^{\circ}$ 647383: SPIRE with PI Mathis) and from the KULeuven Research Council (grant C16/18/005: PARADISE). TVR is funded by the Research Foundation Flanders (FWO) by means of a Junior Postdoctoral Fellowship under grant agreement $\mathrm{N}^{\circ} 12 \mathrm{ZB} 620 \mathrm{~N}$. VP and SM acknowledge support from the CNES PLATO grant at CEA/DAP. 


\section{References}

Aerts, C. 2021, Rev. Mod. Phys., in press, arXiv:1912.12300

Aerts, C., Mathis, S., \& Rogers, T. M. 2019, ARA\&A, 57, 35

Aerts, C., Molenberghs, G., Michielsen, M., et al. 2018, ApJS, 237, 15

Asplund, M., Grevesse, N., Sauval, A. J., \& Scott, P. 2009, ARA\&A, 47, 481

Auvergne, M., Bodin, P., Boisnard, L., et al. 2009, A\&A, 506, 411

Berthomieu, G., Gonczi, G., Graff, P., Provost, J., \& Rocca, A. 1978, A\&A, 70, 597

Borucki, W., Koch, D., Batalha, N., et al. 2009, in IAU Symposium, Vol. 253 Transiting Planets, ed. F. Pont, D. Sasselov, \& M. J. Holman, 289-299

Bouabid, M. P., Dupret, M. A., Salmon, S., et al. 2013, MNRAS, 429, 2500

Boyd, J. P. 1976, PhD thesis, Harvard University

Christophe, S., Ballot, J., Ouazzani, R. M., Antoci, V., \& Salmon, S. J. A. J. 2018, A\&A, 618, A47

Claret, A. \& Torres, G. 2019, ApJ, 876, 134

Deal, M., Richard, O., \& Vauclair, S. 2016, A\&A, 589, A140

Degroote, P., Aerts, C., Baglin, A., et al. 2010, Nature, 464, 259

Dintrans, B. \& Rieutord, M. 2000, A\&A, 354, 86

Dintrans, B., Rieutord, M., \& Valdettaro, L. 1999, Journal of Fluid Mechanics, 398, 271-297

Dziembowski, W. \& Kosovichev, A. 1987a, Acta Astron., 37, 341

Dziembowski, W. \& Kosovichev, A. 1987b, Acta Astron., 37, 313

Dziembowski, W., Kosovichev, A., \& Kozlowski, M. 1987, Acta Astron., 37, 331

Eckart, C. 1960, Hydrodynamics of Oceans and Atmospheres (Pergamon Press (Oxford))

Freytag, B., Ludwig, H. G., \& Steffen, M. 1996, A\&A, 313, 497

Gough, D. O. 1981, MNRAS, 196, 731

Hansen, C. J., Cox, J. P., \& van Horn, H. M. 1977, ApJ, 217, 151

Henyey, L., Vardya, M. S., \& Bodenheimer, P. 1965, ApJ, 142, 841

Herwig, F. 2000, A\&A, 360, 952

Hough, S. S. 1898, Philosophical Transactions of the Royal Society of London Series A, 191, 139

Johnston, C., Aerts, C., Pedersen, M. G., \& Bastian, N. 2019, A\&A, 632, A74

Kaye, A. B., Handler, G., Krisciunas, K., Poretti, E., \& Zerbi, F. M. 1999, PASP, 111,840

Laplace, P. S. 1799, Traité de Mécanique Cëleste (Imprimerie de Crapelet (Paris))

Lee, U. \& Baraffe, I. 1995, A\&A, 301, 419

Lee, U. \& Saio, H. 1986, MNRAS, 221, 365

Lee, U. \& Saio, H. 1987, MNRAS, 224, 513

Lee, U. \& Saio, H. 1997, ApJ, 491, 839

Li, C., Sun, W., de Grijs, R., et al. 2019a, ApJ, 876, 65

Li, G., Bedding, T. R., Murphy, S. J., et al. 2019b, MNRAS, 482, 1757

Li, G., Van Reeth, T., Bedding, T. R., Murphy, S. J., \& Antoci, V. 2019c, MNRAS, 487, 782

Li, G., Van Reeth, T., Bedding, T. R., et al. 2020, MNRAS, 491, 3586

Maeder, A. 2009, Physics, Formation and Evolution of Rotating Stars (Springer A\&A Library)

Maeder, A. \& Zahn, J.-P. 1998, A\&A, 334, 1000

Mathis, S. 2009, A\&A, 506, 811

Mathis, S., Bugnet, L., Prat, V., et al. 2021, A\&A, in press, arXiv:2012.11050

Mathis, S. \& Prat, V. 2019, A\&A, 631, A26

Mathis, S. \& Zahn, J. P. 2004, A\&A, 425, 229

Michielsen, M., Pedersen, M. G., Augustson, K. C., Mathis, S., \& Aerts, C. 2019, A\&A, 628, A76

Miglio, A., Montalbán, J., Noels, A., \& Eggenberger, P. 2008, MNRAS, 386, 1487

Mombarg, J. S. G., Dotter, A., Van Reeth, T., et al. 2020, ApJ, 895, 51

Mombarg, J. S. G., Van Reeth, T., Pedersen, M. G., et al. 2019, MNRAS, 485, 3248

Moravveji, E., Townsend, R. H. D., Aerts, C., \& Mathis, S. 2016, ApJ, 823, 130

Ouazzani, R.-M., Salmon, S. J. A. J., Antoci, V., et al. 2017, MNRAS, 465, 2294

Pápics, P. I., Tkachenko, A., Van Reeth, T., et al. 2017, A\&A, 598, A74

Paxton, B., Cantiello, M., Arras, P., et al. 2013, ApJS, 208, 4

Paxton, B., Smolec, R., Schwab, J., et al. 2019, ApJS, 243, 10

Pedersen, M. G., Aerts, C., Pápics, P. I., \& Rogers, T. M. 2018, A\&A, 614, A128

Prat, V., Mathis, S., Buysschaert, B., et al. 2019, A\&A, 627, A64

Prat, V., Mathis, S., Neiner, C., et al. 2020, A\&A, 636, A100

Ricker, G. R., Winn, J. N., Vanderspek, R., et al. 2014, in Society of PhotoOptical Instrumentation Engineers (SPIE) Conference Series, Vol. 9143, Space Telescopes and Instrumentation 2014: Optical, Infrared, and Millimeter Wave, ed. J. Oschmann, Jacobus M., M. Clampin, G. G. Fazio, \& H. A. MacEwen, 914320

Rogers, T. M. \& McElwaine, J. N. 2017, ApJ, 848, L1

Saio, H., Kurtz, D. W., Murphy, S. J., Antoci, V. L., \& Lee, U. 2018, MNRAS, 474,2774

Shibahashi, H. 1979, PASJ, 31, 87

Sweet, P. A. 1950, MNRAS, 110, 548
Szewczuk, W. \& Daszyńska-Daszkiewicz, J. 2018, MNRAS, 478, 2243

Tassoul, M. 1980, ApJS, 43, 469

Tkachenko, A., Aerts, C., Yakushechkin, A., et al. 2013, A\&A, 556, A52

Tkachenko, A., Pavlovski, K., Johnston, C., et al. 2020, A\&A, 637, A60

Townsend, R. H. D., Goldstein, J., \& Zweibel, E. G. 2018, MNRAS, 475, 879

Townsend, R. H. D. \& Teitler, S. A. 2013, MNRAS, 435, 3406

Unno, W., Osaki, Y., Ando, H., Saio, H., \& Shibahashi, H. 1989, Nonradial oscillations of stars (University of Tokyo Press)

Van Beeck, J., Prat, V., Van Reeth, T., et al. 2020, A\&A, 638, A149

Van Reeth, T., Mombarg, J. S. G., Mathis, S., et al. 2018, A\&A, 618, A24

Van Reeth, T., Tkachenko, A., \& Aerts, C. 2016, A\&A, 593, A120

Van Reeth, T., Tkachenko, A., Aerts, C., et al. 2015, ApJS, 218, 27

Vink, J. S., de Koter, A., \& Lamers, H. J. G. L. M. 2001, A\&A, 369, 574

Waelkens, C. 1991, A\&A, 246, 453

Wang, H., Boyd, J. P., \& Akmaev, R. A. 2016, Geoscientific Model Development, 9,1477

Weiss, W. W., Rucinski, S. M., Moffat, A. F. J., et al. 2014, PASP, 126, 573

Zahn, J.-P. 1966, C. R. Acad. Sci. Paris. Ser. B, 263, 1077

Zahn, J. P. 1992, A\&A, 265, 115 


\section{Appendix A: Centrifugal deformation of Brunt-Väisälä frequency profiles}

After calculating the deformed stellar radius $r(a, \theta)$ using Eqs. (9) and (10), we compute the two-dimensional centrifugally deformed Brunt-Väisälä frequency profile

$N^{2}(a, \theta)=-\frac{\bar{g}}{r}\left[\frac{\mathrm{d} \ln \bar{\rho}}{\mathrm{d} \ln r}-\frac{1}{\bar{\Gamma}_{1}} \frac{\mathrm{d} \ln \bar{P}}{\mathrm{~d} \ln r}\right]$

to ensure numerical stability and to account for the nonlinear dependence of $N^{2}(a, \theta)$ on the density $\bar{\rho}$ and the pressure $\bar{P}$. These calculations are done at discrete values of the co-latitude $\theta$, and we use the calculation methods for $\bar{\rho}$ and $\bar{P}$ described in Section 2.1.1.

The perturbed adiabatic exponent $\bar{\Gamma}_{1}$ can then be estimated as follows:

$$
\begin{aligned}
\frac{1}{\bar{\Gamma}_{1}} & =\left(\frac{\partial \ln \bar{\rho}}{\partial \ln \bar{P}}\right)_{\bar{S}}=\left(\frac{\frac{\partial \ln \bar{\rho}}{\partial a}}{\frac{\partial \ln \bar{P}}{\partial a}}\right)_{\bar{S}} \\
& \approx\left(\frac{\partial \ln \bar{\rho}}{\partial a} \frac{\mathrm{d} \ln P_{0}}{\mathrm{~d} r_{0}}\right)\left(\frac{\mathrm{d} \ln \rho_{0}}{\mathrm{~d} r_{0}} \frac{\partial \ln \bar{P}}{\partial a}\right)^{-1}\left(\frac{\partial \ln \rho_{0}}{\partial \ln P_{0}}\right)_{S} \\
& =\left(\rho_{0} \bar{P} \frac{\partial \bar{\rho}}{\partial a} \frac{\mathrm{d} P_{0}}{\mathrm{~d} r_{0}}\right)\left(\bar{\rho} P_{0} \frac{\mathrm{d} \rho_{0}}{\mathrm{~d} r_{0}} \frac{\partial \bar{P}}{\partial a}\right)^{-1} \frac{1}{\Gamma_{1,0}},
\end{aligned}
$$

where quantities $X$ from the spherical non-rotating input model are indicated as $X_{0}$.

The perturbed gravitational acceleration is the gradient of the effective gravitational potential $\bar{\phi}$;

$\bar{g}=\bar{\nabla} \bar{\phi}=\bar{\nabla}\left(\phi_{0}+\phi_{1}+U\right)=g_{0}+\bar{\nabla}\left(\phi_{1}+U\right)$.

The spheroidal gradient $\bar{\nabla}$, defined as (Mathis \& Prat 2019)

$\bar{\nabla} X \equiv \frac{\partial X}{\partial a} \widetilde{\boldsymbol{e}}_{a}+\frac{1}{a} \frac{\partial X}{\partial \theta} \widetilde{\boldsymbol{e}}_{\theta}+\frac{1}{a \sin \theta} \frac{\partial X}{\partial \varphi} \widetilde{\boldsymbol{e}}_{\varphi}$,

reduces to the total derivative $\mathrm{d} / \mathrm{d} a$ since the calculations of the perturbed quantities are discretized in the co-latitude $\theta$, i.e. they are calculated for a specific, constant value of $\theta$.

To avoid direct numerical differentiation of the perturbed pressure $\bar{P}$, it is rewritten as:

$$
\begin{aligned}
\frac{\mathrm{d} \bar{P}}{\mathrm{~d} r} & =-\bar{\rho} \frac{\mathrm{d} \bar{\phi}}{\mathrm{d} r}=-\frac{\bar{\rho}}{1+4 \varepsilon} \frac{\mathrm{d}}{\mathrm{d} a} \bar{\phi} \\
& =-\frac{\bar{\rho}}{1+4 \varepsilon}\left[g_{0}+\frac{\mathrm{d}}{\mathrm{d} a}\left(\phi_{1}+U\right)\right] \\
& =-\frac{\bar{\rho}}{1+4 \varepsilon}\left[g_{0}+\frac{\mathrm{d}}{\mathrm{d} r_{0}}\left(\phi_{1}+U\right)\right],
\end{aligned}
$$

where we map the radius $r_{0}$ of the non-deformed stellar model onto the pseudo-radius $a$, as in the evaluation of Eq. (11). Within their respective coordinate systems, both $r_{0}$ and $a$ coincide with the isobaric radial coordinate. Consequently, only the last derivative with respect to $r_{0}$ in Eq.(A.9) remains to be evaluated through numerical differentiation. This is numerically more stable than performing the derivation of $\bar{P}$ with respect to $r$. Simi- larly, for the derivative of the perturbed density $\bar{\rho}$ we have:

$$
\begin{aligned}
\frac{\mathrm{d} \bar{\rho}}{\mathrm{d} r}= & \frac{1}{1+4 \varepsilon} \frac{\mathrm{d}}{\mathrm{d} a}\left(\rho_{0}+\rho_{1}\right) \\
= & \frac{1}{1+4 \varepsilon} \frac{\mathrm{d}}{\mathrm{d} r_{0}}\left(\rho_{0}+\rho_{1}\right) \\
= & \frac{1}{1+4 \varepsilon}\left[\frac{\mathrm{d} \rho_{0}}{\mathrm{~d} r_{0}}+\frac{\mathrm{d}}{\mathrm{d} r_{0}}\left(\frac{1}{g_{0}} \frac{\mathrm{d} \rho_{0}}{\mathrm{~d} r_{0}}\left(\phi_{1}+U\right)\right)\right] \\
= & \frac{1}{1+4 \varepsilon} \frac{\mathrm{d} \rho_{0}}{\mathrm{~d} r_{0}}\left[1-\left(\frac{\phi_{1}+U}{g_{0}^{2}}\right) \frac{\mathrm{d} g_{0}}{\mathrm{~d} r_{0}}+\frac{1}{g_{0}} \frac{\mathrm{d}}{\mathrm{d} r_{0}}\left(\phi_{1}+U\right)\right] \\
& +\frac{\phi_{1}+U}{g_{0}(1+4 \varepsilon)} \frac{\mathrm{d}}{\mathrm{d} r_{0}}\left(\frac{\mathrm{d} \rho_{0}}{\mathrm{~d} r_{0}}\right) .
\end{aligned}
$$

The last three derivatives with respect to $a$ are again evaluated through numerical differentiation without numerical issues.

Finally, $\mathrm{d} P_{0} / \mathrm{d} r_{0}$ and $\mathrm{d} \rho_{0} / \mathrm{d} r_{0}$ can be expressed in terms of physical quantities included in the (non-deformed) MESA stellar structure profiles. The former can be calculated by using hydrostatic equilibrium (in a spherically symmetric star):

$\frac{\mathrm{d} P_{0}}{\mathrm{~d} r_{0}}=-\rho_{0} g_{0}$,

and the latter can be retrieved from the non-perturbed squared Brunt-Väisälä frequency:

$\frac{\mathrm{d} \rho_{0}}{\mathrm{~d} r_{0}}=\rho_{0}\left(\frac{1}{\Gamma_{1,0} P_{0}} \frac{\mathrm{d} P_{0}}{\mathrm{~d} r_{0}}-\frac{N_{0}^{2}}{g_{0}}\right)$.

\section{Appendix B: Computation of asymptotic frequencies}

Here we provide our strategy to compute the asymptotic frequencies and period spacing patterns presented in Sect. $3.3 \& 3.4$. This is summarised in a flowchart in Fig. B.1 and concerns the computation of the asymptotic frequencies for given pulsation mode with mode identification $(k, m)$, for a chosen stellar structure model with a particular rotation rate (in terms of $\Omega_{\mathrm{c}}$ ).

The period spacing pattern for a spherically symmetric, uniformly rotating star is computed within a radial order range of $n=1$ to $n=100$. This particular range is motivated by the radial order distributions of typically observed GIWs (Li et al. 2020). Based on the resulting period spacing pattern, a suitable $v$-range is chosen. To anticipate the (relatively small) effect of the centrifugal force on the asymptotic pulsation periods, small margins are taken above and below this range (typically \pm 0.5 ). In the next step, the GLTE is solved for a number of spin parameters within the predetermined range. A step size of 0.01 in $v$ is found suitable to avoid numerical inaccuracies, while maintaining reasonable computation times (typical values for $v_{\max }-v_{\min }$ are on the order of 10). In the subsequent step, the expression for the asymptotic pulsation frequencies Eq. (16) is rewritten as follows:

$\frac{1}{\omega_{n k m}}=\frac{(n+1 / 2) \pi}{\int_{a_{1}}^{a_{2}} \frac{\Lambda_{v k m}^{1 / 2}(a) \bar{N}(a)}{a} \mathrm{~d} a}$,

and multiplied by $2 \Omega$ to arrive at:

$I(v)=v \int_{a_{1}}^{a_{2}} \frac{\Lambda_{v k m}^{1 / 2}(a) \bar{N}(a)}{a} \mathrm{~d} a=2 \Omega(n+1 / 2) \pi$. 


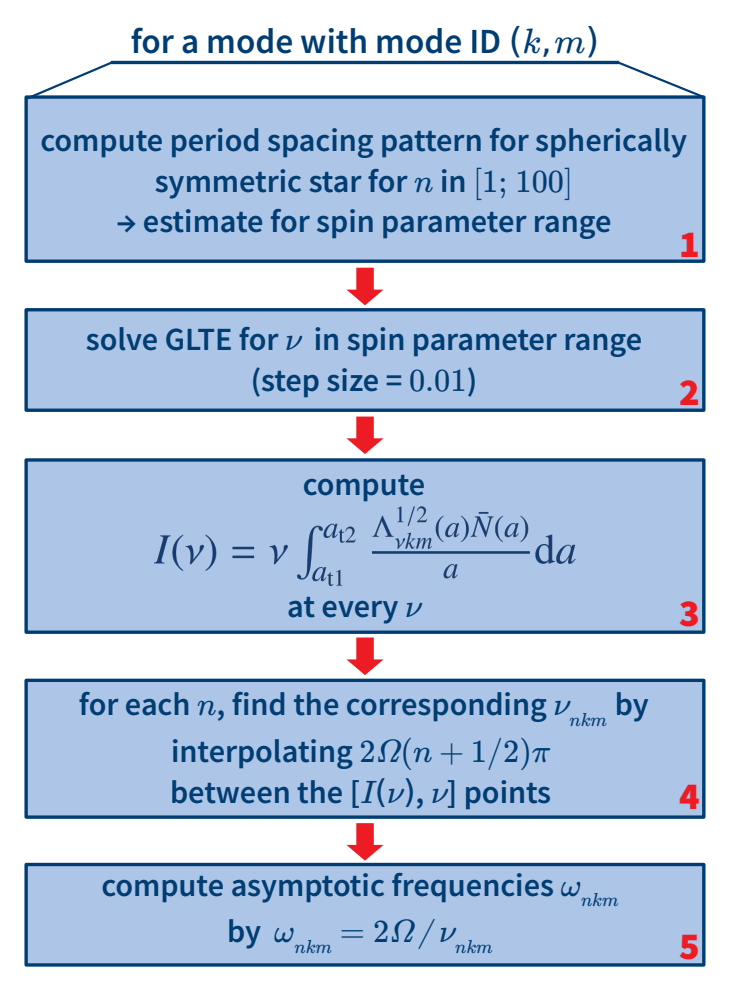

Fig. B.1. Flowchart describing the strategy used in this work to compute asymptotic pulsation frequencies in a centrifugally deformed star.

The LHS of this equation is then evaluated at every $v$ in the spin parameter range, while the RHS is evaluated for every $n \in[1 ; 100]$. In the fourth step, the spin parameters $v_{n k m}$ corresponding to the radial orders $n \in[1 ; 100]$ are computed through linear interpolation of the RHS of Eq. (B.2) to the $(I(v), v)$-points from the previous step. Finally, the asymptotic angular frequencies $\omega_{n k m}$ are retrieved by taking the inverse of the $v_{n k m}$ from the previous step, and multiplying them with $2 \Omega$. The corresponding asymptotic pulsation periods are $P_{n k m}=2 \pi / \omega_{n k m}$. The corresponding pulsation periods in the inertial (observer's) frame $P_{n k m}^{\text {in }}$ are then found through (Bouabid et al. 2013):

$P_{n k m}^{\mathrm{in}}=\frac{P_{n k m}}{1+m \frac{P_{n k m}}{P_{\mathrm{rot}}}}$,

with $P_{\text {rot }}$ the rotation period.

\section{Appendix C: Comparison with other theoretical assumptions}

Other assumptions that are included within the TAR, are still made in the generalised framework developed by Mathis \& Prat (2019), such as the neglect of the horizontal rotation vector component $\boldsymbol{\Omega}_{\mathrm{H}}$ and the Cowling approximation. Here we assess the impact of these approximations on our results.

\section{Appendix C.1: Neglect of the horizontal rotation vector}

The spheroidal radial component of the momentum equation is given by Mathis \& Prat (2019, Eq.(13)) as

$$
\begin{aligned}
-\bar{N}^{2}\left(\frac{\omega}{\bar{N}}\right)^{2}\left[\left(1+2\left(\varepsilon+a \partial_{a} \varepsilon\right)\right) \xi_{a}+\xi_{\theta} \partial_{\theta} \varepsilon\right] \\
-i \bar{N}^{2}\left(\frac{\omega}{\bar{N}}\right)\left(\frac{2 \Omega}{\bar{N}}\right)\left(1+2 \varepsilon+a \partial_{a} \varepsilon\right) \sin \theta \xi_{\varphi} \\
=-\partial_{a} \widetilde{W}-\bar{N}^{2} \xi_{a}-\frac{1}{\bar{\rho}^{2}} \partial_{a} \bar{\rho} \widetilde{P}
\end{aligned}
$$

where $\widetilde{W}=\widetilde{P} / \bar{\rho}$ with $\widetilde{P}$ the wave fluctuation of the pressure, and $\boldsymbol{\xi}=\left(\xi_{a}, \xi_{\theta}, \xi_{\phi}\right)$ is the Lagrangian displacement vector. Within the generalised TAR-framework, the two terms on the left-hand side (LHS) of Eq.(C.1) are neglected in favour of the $\bar{N}^{2} \xi_{a}$-term on the right-hand side (RHS), and the second LHS-term contains the horizontal component of the rotation vector $\Omega_{\mathrm{H}}=\Omega \sin \theta$. Mathis \& Prat (2019) combined the simplified Eq.(C.1) with the horizontal components of the momentum equation, and solved the resulting system for a selected pulsation mode identification $(k, m)$ and spin parameter value $v$ as a function of the normalised pressure $W_{v k m}^{\prime}(a, \theta)$ :

$\xi_{a ; v k m}^{\prime}(a, \theta)=-i k_{V ; v k m}(a) \frac{W_{v k m}^{\prime}}{\bar{N}^{2}(a)}=-i \frac{\sqrt{\Lambda_{v k m}(a)}}{a \omega_{k m}} \frac{W_{v k m}^{\prime}}{\bar{N}(a)}$
$\xi_{\theta ; v k m}^{\prime}(a, \theta)=\frac{1}{a} \frac{1}{\omega_{k m}^{2}} \frac{1}{\mathcal{D}}\left[\partial_{\theta} W_{v k m}^{\prime}-m \nu \frac{\cos \theta}{\sin \theta} C W_{v k m}^{\prime}\right]$
$\xi_{\varphi ; v k m}^{\prime}(a, \theta)=i \frac{1}{a} \frac{1}{\omega_{k m}^{2}} \frac{1}{\mathcal{D}}\left[\nu C \cos \theta \partial_{\theta} W_{v k m}^{\prime}-\frac{m}{\sin \theta} W_{v k m}^{\prime}\right]$

Expressions for the coefficients $C(a, \theta)$ and $D(a, \theta)$ are given in Eqs.(20) to (25).

We assess the validity of neglecting the LHS-terms in Eq.(C.1) by taking their ratios with the $\bar{N}^{2} \xi_{a}$-term and filling in the solution for $\xi_{v k m}^{\prime}$. For the first LHS-term, we obtain

$$
\begin{aligned}
\bar{N}^{2}\left(\frac{\omega_{k m}}{\bar{N}}\right)^{2}\left[\left(1+2\left(\varepsilon+a \partial_{a} \varepsilon\right)\right) \xi_{a ; v k m}+\xi_{\theta ; v k m} \partial_{\theta} \varepsilon\right]\left[\bar{N}^{2} \xi_{a ; v k m}\right]^{-1} \\
\simeq\left(\frac{\omega_{k m}}{\bar{N}}\right)^{2}(1+8 \varepsilon)+\left(\frac{\omega_{k m}}{\bar{N}}\right) \frac{3 \varepsilon_{l=2}}{\mathcal{D} \sqrt{\Lambda_{v k m}}} \\
\times\left[\frac{\sin 2 \theta \partial_{\theta} W_{v k m}^{\prime}}{2 i W_{v k m}^{\prime}}-\frac{m v C \cos ^{2} \theta W_{v k m}^{\prime}}{i W_{v k m}^{\prime}}\right],
\end{aligned}
$$

and for the second LHS-term, we find

$$
\begin{aligned}
& i \bar{N}^{2}\left(\frac{\omega_{k m}}{\bar{N}}\right)\left(\frac{2 \Omega}{\bar{N}}\right)\left(1+2 \varepsilon+a \partial_{a} \varepsilon\right) \sin \theta \xi_{\varphi}\left[\bar{N}^{2} \xi_{a, v k m}\right]^{-1} \\
& \simeq\left(\frac{2 \Omega}{\bar{N}}\right) \frac{(1+5 \varepsilon)}{2 \mathcal{D} \sqrt{\Lambda_{v k m}}}\left[\sin (2 \theta) \nu C \frac{\partial_{\theta} W_{v k m}^{\prime}}{i W_{v k m}^{\prime}}-\frac{2 m W_{v k m}^{\prime}}{i W_{v k m}^{\prime}}\right] .
\end{aligned}
$$

Hence, the errors introduced by neglecting the terms in Eq.C.1, scale with $\omega_{k m} / \bar{N}$ and $2 \Omega / \bar{N}$, respectively. We can ignore the contribution from the horizontal component of the rotation vector when the GIWs propagate in a strongly stratified radiative region, i.e., for $2 \Omega \ll \bar{N}$. This is further illustrated in Fig. C.1, where we compare the terms in the momentum equation Eq.(C.1) for a $(n, k, m)=(50,0,1)$ pulsation mode in the central model of our MESA grid, with a rotation rate $\Omega=$ $0.15 \Omega_{\mathrm{c}}$. The relative contribution of the neglected LHS-terms, which include the $\Omega \sin \theta$-component of the rotation vector, is $~$ $1 \%$. The (normalised) components of the Lagrangian displacement $\left(\xi_{a}, \xi_{\theta}, \xi_{\phi}\right)$, calculated in this simulation using Eqs. (C.2) to (C.4) are shown in Fig. C.2. As can be seen, $\xi_{a} \ll \xi_{\theta}, \xi_{\varphi}$. 

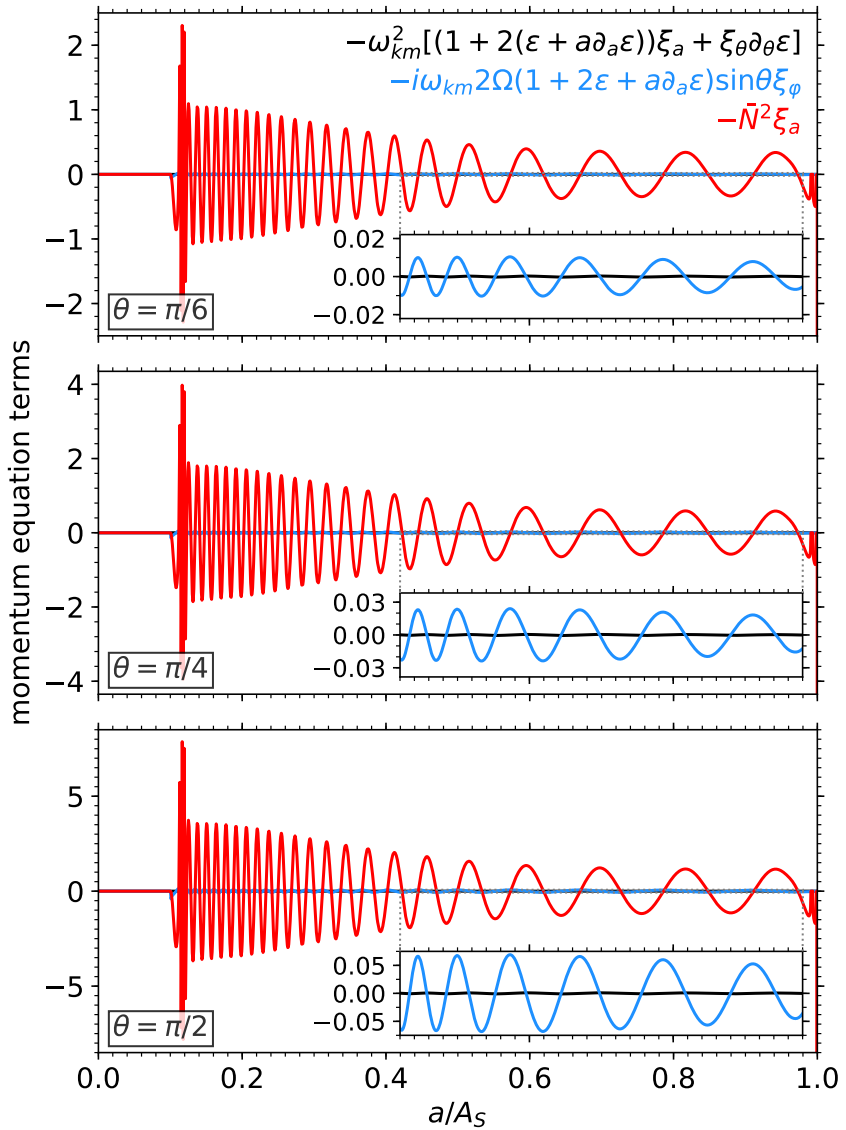

Fig. C.1. The $\bar{N}^{2} \xi_{a}$-term (red), the first LHS-term (black) and second LHS-term (blue) in the momentum equation Eq.(C.1), calculated for the $(n, k, m)=(50,0,1)$ pulsation mode in the central model of our MESA grid, with $2 \mathrm{M}_{\odot}, X_{\mathrm{c}}=0.50$ and a rotation rate $\Omega=0.15 \Omega_{\mathrm{c}}$. The values are shown at co-latitudes $\theta=\pi / 6$ (top), $\pi / 4$ (middle) and $\pi / 2$ (bottom). Parts of the LHS-terms are shown again in the insets, magnified $27 \times$.

\section{Appendix C.2: The Cowling approximation}

We evaluate the Cowling approximation for the central MESA model in our grid $\left(2 \mathrm{M}_{\odot}, X_{\mathrm{c}}=0.50\right)$ using GYRE, for rotation rates $\Omega / \Omega_{\mathrm{c}} \in[0.10-0.70]$, without the centrifugal acceleration. As shown in Fig. C.3, the relative differences between the pulsation frequencies, caused by the Cowling approximation, are $\propto 0.1 \%$. These are smaller than the relative differences introduced by the centrifugal acceleration, but increase with increasing radial order of the $g$ modes.

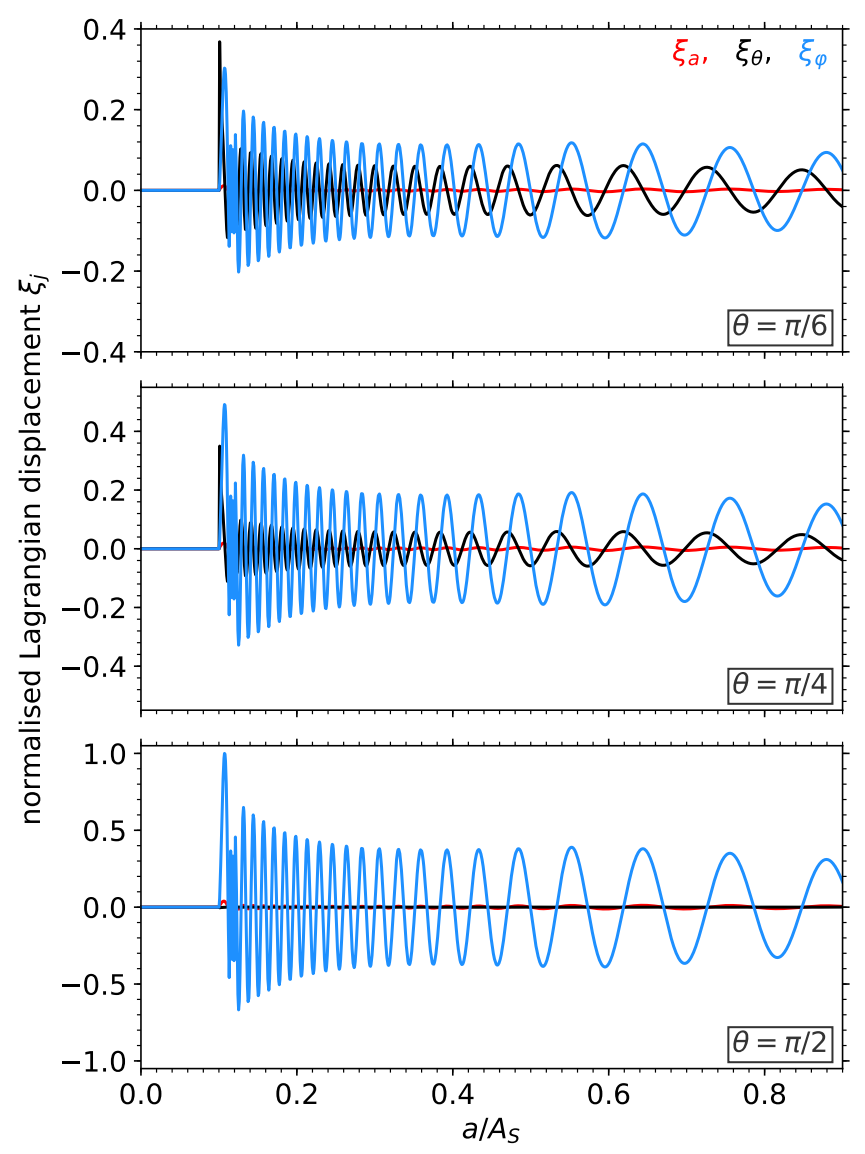

Fig. C.2. The (normalised) components of the Lagrangian displacement $\xi_{a}$ (red), $\xi_{\theta}$ (black) and $\xi_{\phi}$ (blue), calculated in the numerical simulations shown in Fig. C.1. The functions are shown at co-latitudes $\theta=\pi / 6$ (top), $\pi / 4$ (middle) and $\pi / 2$ (bottom).

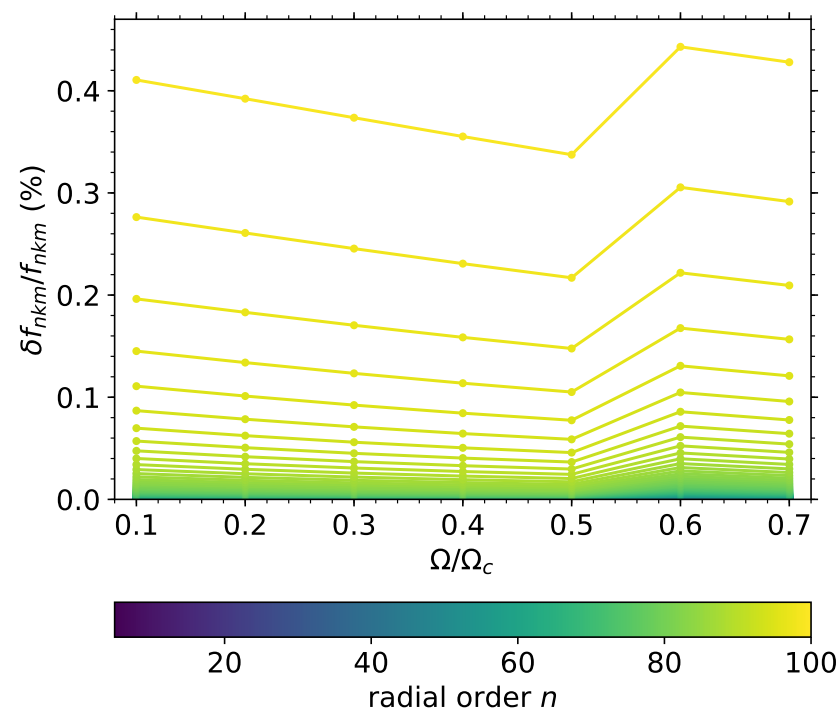

Fig. C.3. Relative pulsation frequency shifts $\delta f_{n k m} / f_{n k m}$ caused by the Cowling approximation, with $\delta f_{n k m}=f_{\text {Cowling }}-f_{\text {withoutCowling }}$ and $f_{n k m}=$

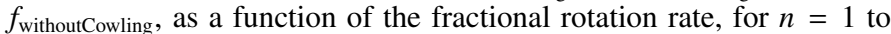
$n=100$. Calculations are done using the central $2 M_{\odot}, X_{c}=0.50 \mathrm{MESA}$ model with $\Omega / \Omega_{\mathrm{c}} \in[0.10-0.70]$, for prograde dipole sectoral $(k=$ $0, m=1$ ) modes. 